\title{
The $\gamma$-Protocadherins Regulate the Survival of GABAergic Interneurons during Developmental Cell Death
}

\author{
Candace H. Carriere, ${ }^{1 *}{ }^{\circ}$ Wendy Xueyi Wang, ${ }^{1,2 *}{ }^{(}$Anson D. Sing, ${ }^{1 *}$ Adam Fekete, ${ }^{1}$ Brian E. Jones, ${ }^{1}$ \\ ${ }^{\circ}$ Yohan Yee, ${ }^{3,5}{ }^{10}$ Jacob Ellegood, ${ }^{5}$ Harinad Maganti, ${ }^{1}$ Lola Awofala, ${ }^{1}$ Julie Marocha, ${ }^{1,2}$ Amar Aziz, ${ }^{1}$ \\ Lu-Yang Wang, ${ }^{1,4}$ Jason P. Lerch, ${ }^{3,5,6}$ and ${ }^{\circledR}$ Julie L. Lefebvre ${ }^{1,2}$ \\ ${ }^{1}$ Program for Neuroscience and Mental Health, Hospital for Sick Children, Toronto, Ontario M5G 0A4, Canada, ${ }^{2}$ Department of Molecular Genetics, \\ University of Toronto, Ontario M5S 1A8, Canada, ${ }^{3}$ Department of Medical Biophysics, University of Toronto, Toronto, Ontario M5G 1L7, Canada, \\ ${ }^{4}$ Department of Physiology, University of Toronto, Toronto, Ontario M5S 1A8, Canada, ${ }^{5}$ Mouse Imaging Centre, Hospital for Sick Children, \\ Toronto, Ontario M5T 3H7, Canada, and ${ }^{6}$ Wellcome Centre for Integrative Neuroimaging, FMRIB, Nuffield Department of Clinical Neuroscience, \\ University of Oxford, Oxford, United Kingdom, OX3 9DU
}

Inhibitory interneurons integrate into developing circuits in specific ratios and distributions. In the neocortex, inhibitory network formation occurs concurrently with the apoptotic elimination of a third of GABAergic interneurons. The cell surface molecules that select interneurons to survive or die are unknown. Here, we report that members of the clustered Protocadherins ( $\mathrm{cPCDHs}$ ) control GABAergic interneuron survival during developmentally-regulated cell death. Conditional deletion of the gene cluster encoding the $\gamma$-Protocadherins (Pcdhgs) from developing GABAergic neurons in mice of either sex causes a severe loss of inhibitory populations in multiple brain regions and results in neurologic deficits such as seizures. By focusing on the neocortex and the cerebellar cortex, we demonstrate that reductions of inhibitory interneurons result from elevated apoptosis during the critical postnatal period of programmed cell death (PCD). By contrast, cortical interneuron (cIN) populations are not affected by removal of Pcdhgs from pyramidal neurons or glial cells. Interneuron loss correlates with reduced AKT signaling in Pcdhg mutant interneurons, and is rescued by genetic blockade of the pro-apoptotic factor BAX. Together, these findings identify the PCDHGs as pro-survival transmembrane proteins that select inhibitory interneurons for survival and modulate the extent of PCD. We propose that the PCDHGs contribute to the formation of balanced inhibitory networks by controlling the size of GABAergic interneuron populations in the developing brain.

Key words: apoptosis; cortical development; GABAergic interneuron; inhibitory neurons; programmed cell death; Protocadherins

Significance Statement

A pivotal step for establishing appropriate excitatory-inhibitory ratios is adjustment of neuronal populations by cell death. In the mouse neocortex, a third of GABAergic interneurons are eliminated by BAX-dependent apoptosis during the first postnatal week. Interneuron cell death is modulated by neural activity and pro-survival pathways but the cell-surface molecules that select interneurons for survival or death are unknown. We demonstrate that members of the cadherin superfamily, the clustered $\gamma$-Protocadherins (PCDHGs), regulate the survival of inhibitory interneurons and the balance of cell death. Deletion of the Pcdhgs in mice causes inhibitory interneuron loss in the cortex and cerebellum, and leads to motor deficits and seizures. Our findings provide a molecular basis for controlling inhibitory interneuron population size during circuit formation.

Received June 25, 2020; revised Sep. 23, 2020; accepted Sep. 29, 2020.

Author contributions: J.L.L., L.Y.W., Y.Y., J.E., J.P.L., A.F., C.H.C., A.D.S., W.X.W., B.E.J., and H.M. designed research; J.L.L., J.M., Y.Y., J.E., A.F., C.H.C., A.D.S., W.X.W., B.E.J., H.M., L.A., and A.A. performed research; J.L.L., Y.Y., J.E., J.P.L., A.F., C.H.C., A.D.S., W.X.W., and B.E.J. analyzed data; J.L.L. wrote the paper.

${ }^{*}$ C.H.C., W.X.W., and A.D.S. contributed equally to this work.

This work was supported by the Scottish Rite Charitable Foundation of Canada Award 16107, a Canada Research Chair Tier 2, a Sloan Fellowship in Neuroscience, and the Canadian Institutes of Health Research (CIHR) Project Grant PJT-148961 (to J.L.L.); a Canada Research Chair Tier 1 and CIHR Project Grants PJT156034 and PJT-156439 (to L-Y.W.); a University of Toronto Open Fellowship and a Restracomp PhD Student award (J.M.); the Natural Sciences and Engineering Research Council of Canada Undergraduate Research
(A.A.); a Hospital for Sick Children Restracomp Postdoctoral Fellowship (A.D.S.); and by the Province of Ontario Neurodevelopmental Disorders Programme from the Ontario Brain Institute (J.P.L.).

The authors declare no competing financial interests.

Acknowledgements: We thank Dr. Krutika Joshi and Dr. Miguel Cortez (SickKids) for assistance for EEG recordings and analyses. We also thank members of the Lefebvre laboratory for comments on this manuscript.

Correspondence should be addressed to Julie L. Lefebvre at julie.lefebvre@sickkids.ca.

https://doi.org/10.1523/JNEUROSCI.1636-20.2020

Copyright $\odot 2020$ the authors 


\section{Introduction}

The optimal function of neuronal networks is dependent on establishing appropriate densities of GABA-releasing interneurons that provide specialized forms of synaptic inhibition to shape principal neuron firing and network synchronization. One mechanism that adjusts neuron population sizes is programmed cell death (PCD). The importance of PCD for inhibitory interneurons was demonstrated recently in the mouse neocortex, where $30-40 \%$ of GABAergic interneurons are eliminated between postnatal day (P)5 and P10 (Southwell et al., 2012). Cortical interneuron (cIN) apoptosis occurs on the heels of pyramidal PCD through the BAX-dependent intrinsic pathway and affects the major cIN classes deriving from the ganglionic eminences (Verney et al., 2000; Southwell et al., 2012; Blanquie et al., 2017; Denaxa et al., 2018; Priya et al., 2018; Wong et al., 2018).

The final determination of interneuron population sizes requires pro-survival factors to control the extent of PCD. Neural activity is one factor that influences PCD in the cortex by modulating pro-survival pathways such as PI3K/AKT and Calcineurin/NFAT (Hardingham et al., 2002; Wagner-Golbs and Luhmann, 2012; Priya et al., 2018; Wong et al., 2018) and suppressing pro-apoptotic effectors (Heck et al., 2008; Léveillé et al., 2010; Golbs et al., 2011). Blocking cIN activity by NMDA antagonists or chemogenetic approaches accentuates cIN apoptotic loss while depolarization increases the number of surviving interneurons (Wang et al., 2017; Denaxa et al., 2018; Priya et al., 2018). However, the influence of activity is cIN class specific (Priya et al., 2018), and cell-intrinsic or population-intrinsic factors also contribute to cIN survival (Southwell et al., 2012). Lifeor-death decisions among developing GABAergic interneurons are regulated by multiple interactions but the molecules that mediate them are unknown.

A major regulator of interneuron survival in the spinal cord and retina are the clustered Protocadherins (cPCDHs; Wang et al., 2002; Weiner et al., 2005; Lefebvre et al., 2008; Prasad et al., 2008). In mice, the cPCDHs comprise 58 cadherin-related transmembrane molecules encoded by three tandemly-arranged gene clusters, Pcdh- $\alpha$ (Pcdha), Pcdh- $\beta$ (Pcdhb), and Pcdh- $\gamma$ (Pcdhg; $\mathrm{Wu}$ and Maniatis, 1999). Noted for their immense cell surface diversity and homophilic selectivity (Thu et al., 2014; Mountoufaris et al., 2018), the cPCDHs play diverse roles in neurite patterning such as arborization and self-avoidance (Katori et al., 2009; Garrett et al., 2012; Lefebvre et al., 2012; Molumby et al., 2016; Chen et al., 2017a; Lefebvre, 2017; Mountoufaris et al., 2017; Ing-Esteves et al., 2018). The cPCDHs also have an essential but cell type-specific role in regulating developmental, BAX-dependent PCD. Constitutive Pcdh- $\gamma$ mutants die at birth from massive apoptosis in the hindbrain and spinal cord (Wang et al., 2002; Weiner et al., 2005). Conditional Pcdhg mutant retinas and spinal cords exhibit a disproportionate loss of interneurons to apoptosis (Lefebvre et al., 2008; Prasad et al., 2008). While sensory neurons and principal motor neurons are spared, retinal ganglion cells are affected, suggesting that PCDHG-dependent survival is not exclusive to interneurons. Of the 22 Pcdhgs, retinal and spinal interneuron survival requires the three C-type Pcdhgc3-c5 (Chen et al., 2012), and as shown recently, the single Pcdhgc4 gene is essential (Garrett et al., 2019). While Pcdha and Pcdhb mutant mice exhibit little to no cell loss, neuronal death phenotypes are profoundly exacerbated in double Pcdha; Pcdhg mutant retinas and triple $c P c d h$ knock-out spinal cords, revealing complex redundancy (Hasegawa et al., 2016; IngEsteves et al., 2018). The pro-survival and pro-death pathways regulated by the $\mathrm{CPCDH}$ are unknown.
In the brain, the $c P c d h$ s are dispensable for the survival of principal neuron populations such as cortical pyramidal neurons, Purkinje cells, and serotonergic projection neurons (Katori et al., 2009; Garrett et al., 2012; Lefebvre et al., 2012; Chen et al., 2017a). Using conditional Pcdhg mice and GABAergic Cre drivers, we asked whether the PCDHGs regulate inhibitory interneuron survival. Focusing on interneuron populations in the cortex and cerebellum, we demonstrate that the PCDHGs mediate a pro-survival mechanism that modulates AKT signaling for the specific control of inhibitory interneuron survival during developmentally-regulated cell death.

\section{Materials and Methods}

Mouse strains

Mouse lines used in this study have been described previously. The Pcdhg ${ }^{f c o n} 3$ conditional allele contains loxP sequences that flank the third constant exon and generates a functionally null allele following Cre recombination (Lefebvre et al., 2008; Prasad et al., 2008). The Gad2-irescre knock-in line (JAX \#028867) co-expresses Cre with Gad2/GAD65 in developing GABAergic neurons and a small percentage of cortical astrocytes (Taniguchi et al., 2011; Taniguchi, 2014). Constitutive and conditional floxed Bax alleles were used for the cerebellar and neocortical studies, respectively (constitutive Bax ${ }^{\text {tm1Sjk }}$, JAX \#002994; floxed Bax ${ }^{\text {tm2Sjk }}$, JAX \#006329; Knudson et al., 1995; Takeuchi et al., 2005). In contrast to the available Bax $x^{\text {tm2Sjk }} B a k 1^{\text {tmlThsn }}$ mice, the knock-out Bak allele was not maintained with $B a x^{f / f}$ for this study. The following lines were used: Emx1-ires-cre (JAX \#005628; Gorski et al., 2002); Nkx2.1-cre BAC transgenic (JAX \#008661; Xu et al., 2008); PV-ires-cre (Pvalbtm1 $(\mathrm{cre})^{\text {Arbr/J}}$; JAX \#017320; Hippenmeyer et al., 2005); the Cre reporter line Ai14-Rosa26 $6^{\text {lsl-tdTomato }}$ (herein referred to as Ail4-TdTomato; JAX \#007914; Madisen et al., 2010). Mice were maintained on a C57/B6J background. Wild-type and/or heterozygous Pcdhg; Cre-positive siblings of either sex were selected for the control cohorts. As cortical cIN densities did not differ between wild-type and heterozygous siblings, data were pooled as controls, as indicated in the text. Animals were coded to blind the investigators of the genotypes.

Animal experiments were performed in accordance with the Canadian Council on Animal Care Guidelines for Use of Animals in Research and Laboratory Animal Care under protocols approved by The Centre for Phenogenomics Animal Care Committee and the Laboratory of Animal Services Animal Use and Care Committee at the Hospital for Sick Children (Toronto, Canada).

\section{Electroencephalogram (EEG) studies}

Animals were anesthetized using isoflurane ( $4 \%$ in $\mathrm{O}_{2}$ for induction and $1.5-2 \%$ in $\mathrm{O}_{2}$ for maintenance) in a stereotaxic apparatus, where they were given $1 \mathrm{ml}$ of $0.9 \%$ saline and $5 \mathrm{mg} / \mathrm{kg}$ Anafen for analgesia. Tento 12-week-old mice were implanted with a two-tungsten microelectrode with polyimide tubing, with one probe placed into the frontal cortical regions and the reference electrode in the parietal cortex (wire diameter, $125 \mu \mathrm{m}$; length, $400 \mu \mathrm{m}$, MicroProbes). Electrodes were secured using cyanoacrylate (Krazy Glue) and Ortho-Jet acrylic (Central Dental Ltd.). All animals had a minimum postoperative recovery period of $3 \mathrm{~d}$ before recordings. EEG activity was recorded in freely-moving animals for 90 min using a Grass amplifier and software (Grass Instruments) and monitored by a videorecorder. Mice were placed in warm Plexiglas chambers (Harvard Apparatus) for 1-h acclimation period (Joshi et al., 2018). Brains were harvested to verify electrode placement. EEG recordings were obtained and analyzed from the bandwidth of $1-\mathrm{Hz}$ low-frequency filter (LFF) and 70-Hz high-frequency filter (HFF) and with the $60-\mathrm{Hz}$ notch filter "ON." For Racine scale assessment with EEG confirmation, video episodes lasting $25 \mathrm{~min}$ during which animals exhibited extended immobility were selected. The number and time lengths of seizure episodes were quantified and assessed by a modified Racine scale: (1) stiffness and rigid posturing; (2) head nodding and tilting; (3) partial forelimb clonus; (4) whole body seizures; (5) motor convulsions, including falling, forelimb clonus and jumping (Racine, 1972; Kim et al., 2019). 
Slice preparation and electrophysiology

Acute coronal cortical slices $(300 \mu \mathrm{m})$ containing visual cortex were prepared from P13-P15 Pcdhg ${ }^{W T}$ or Het; Gad2-Cre; Ai14-TdTomato and Pcdhg $g^{f f f}$;Gad2-Cre; Ai14-TdTomato littermates of either sex. After decapitation the brain was placed into ice-cold cutting solution containing the following: $87 \mathrm{~mm} \mathrm{NaCl}, 75 \mathrm{~mm}$ sucrose, $2.5 \mathrm{~mm} \mathrm{KCl}, 10 \mathrm{~mm}$ glucose, 1.25 $\mathrm{mm} \mathrm{NaH} \mathrm{PO}_{4}, 2 \mathrm{~mm}$ Na-pyruvate, 3 mм myo-inositol, $0.5 \mathrm{~mm}$ ascorbic acid, $26 \mathrm{~mm} \mathrm{NaHCO}_{3}, 7 \mathrm{~mm} \mathrm{MgCl}_{2}$, and $0.1 \mathrm{~mm} \mathrm{CaCl}_{2}$ (pH 7.4) and sliced on a vibratome (VT1200S, Leica). Slices were transferred into a holding chamber containing the cutting solution, incubated for $30 \mathrm{~min}$ at $35^{\circ} \mathrm{C}$ then stored at room temperature until use. For electrophysiology experiments, slices were continuously perfused $(2 \mathrm{ml} / \mathrm{min})$ at room temperature with artificial cerebrospinal fluid containing the following: 125 $\mathrm{mm} \mathrm{NaCl}, 2.5 \mathrm{~mm} \mathrm{KCl}, 10 \mathrm{~mm}$ glucose, $1.25 \mathrm{~mm} \mathrm{NaH}_{2} \mathrm{PO}_{4}, 2$ mм Na-pyruvate, $3 \mathrm{~mm}$ myo-inositol, $0.5 \mathrm{~mm}$ ascorbic acid, $26 \mathrm{~mm} \mathrm{NaHCO}_{3}, 1 \mathrm{~mm}$ $\mathrm{MgCl}_{2}$, and $2 \mathrm{~mm} \mathrm{CaCl}$ ( $\mathrm{pH}$ 7.4). All extracellular solutions were oxygenated with $95 \% \mathrm{O}_{2}$ and $5 \% \mathrm{CO}_{2}$. Layer $\mathrm{V}$ cINs were visualized by an upright microscope (Axio Examiner, Carl Zeiss) equipped with a CCD monochrome video camera (IR-1000, DAGE-MTI) and identified by their localization in Layer $\mathrm{V}$ and TdTomato expression. TdTomato was excited using a mercury-lamp (X-cite 120PC, EXFO Photonic Solution Inc.) and an appropriate filter set (BP545/25ex, FT570 beam splitter, BP605/70em). Cells with swollen somata and blebs on their processes were discarded. Electrophysiology data were sampled $(50 \mathrm{kHz})$ and lowpass filtered ( $4 \mathrm{kHz}$ four-pole Bessel filter) by a Multiclamp 700B dual channel amplifier (Molecular Devices). For whole-cell current-clamp recordings 4- to $5-\mathrm{M} \Omega$ pipettes were pulled (PP-830, Narishige) from borosilicate glass with filament (WPI) and filled with the following: 122 mM K-gluconate, $8 \mathrm{~mm} \mathrm{KCl}, 0.5 \mathrm{~mm}$ EGTA, $10 \mathrm{~mm}$ HEPES, $1 \mathrm{~mm}$ $\mathrm{MgCl}_{2}, 2 \mathrm{~mm} \mathrm{Na}$-ATP, $0.5 \mathrm{~mm}$ Na-GTP, $10 \mathrm{~mm}$ phosphocreatine di(tris) salt, and $0.12 \mathrm{~mm}$ Alexa Fluor 488 hydrazide, $\mathrm{pH}$ adjusted to 7.3 with $\mathrm{KOH}$. After forming $>1 \mathrm{G} \Omega$ resistance seal in cell-attached configuration, we broke the patch membrane and kept the neurons at $-70 \mathrm{mV}$. Voltages were not corrected for liquid junction potential. Series resistances were $<25 \mathrm{M} \Omega$ and compensated by the bridge balance circuitry in current-clamp recordings. Cells requiring holding current $>-150 \mathrm{pA}$ at $-70 \mathrm{mV}$ were discarded. The resting membrane potential (Vrest) was tested in $\mathrm{I}=0$ configuration. Intrinsic excitability was tested by $600-\mathrm{ms}-$ long current steps between 200 and +500 pA with 50-pA increments in the presence of AMPA-receptor, NMDA-receptor, and $\mathrm{GABA}_{\mathrm{a}}$-receptor blockers (AP-5, $50 \mu \mathrm{M}$; NBQX, $10 \mu \mathrm{M}$; picrotoxin, $100 \mu \mathrm{M}$, respectively). Input resistance (Rin) was quantified using a small hyperpolarizing step $(-50 \mathrm{pA})$. Data were analyzed offline by pCLAMP 10 software package (Molecular Devices, RRID:SCR_011323) and Excel (Microsoft).

\section{Magnetic resonance imaging (MRI)}

Mice were transcardially perfused and fixed with $4 \%$ paraformaldehyde (PFA) containing $2 \mathrm{~mm}$ Prohance, and brains were prepared as described previously (Allemang-Grand et al., 2017). A 7.0-Tesla MRI scanner (Agilent Ince.) was used to acquire all images, as described previously (Lerch et al., 2011; Cahill et al., 2012; de Guzman et al., 2016). To detect volume differences throughout the brain, we used the following parameters for the MRI scan that produce images with $0.040-\mathrm{mm}$ isotropic voxels: T2-weighted, 3-D fast spin-echo sequence with a cylindrical acquisition of k-space, a TR of $350 \mathrm{~ms}$, and TEs of $12 \mathrm{~ms}$ per echo for six echoes, field-of-view equaled to $20 \times 20 \times 25 \mathrm{~mm}^{3}$, and matrix size equaled to $504 \times 504 \times 630$. Deformation-based morphometry $(\mathrm{DBM})$ was used to register the images and analyze the volume differences following linear and nonlinear analyses using mni_autoreg tools and ANTS (advanced normalization tools; Avants et al., 2008, 2011). Scans were resampled with the appropriate transform and averaged to create a population atlas representing the average anatomy of the study sample. The result of the registration is to have all images deformed into alignment with each other in an unbiased fashion, and to model how the deformation fields relate to genotype (Lerch et al., 2008). Significant regional volume differences are calculated by warping a preexisting classified MRI atlas onto the population atlas, and allowing for assessment of 182 different segmented structures (Dorr et al., 2008; Steadman et al., 2014).
Multiple comparisons were controlled for using the false discovery rate (FDR; Genovese et al., 2002).

\section{Spatial gene expression correlates of brain volume changes}

We employed the adult and developing mouse brain in situ hybridization datasets from the Allen Institute's Mouse Brain Atlas and the Developing Mouse Brain Atlas (Lein et al., 2007; Sunkin et al., 2013). Data describing the spatial expression of Gad2 mRNA were acquired at embryonic day (E)18.5 and P56 in terms of "gene expression energy," which defines the sum of the expressed gene pixel intensities in a given brain region divided by the total number of pixels in that region. In the case of the E18.5 data, the Allen Institute's REST API was queried to mine regional expression energy data for brain regions described in their Developing Mouse Brain Atlas ontology (ontology ID =12). Regional developmental expression of Gad2 was mapped to each voxel on an adult brain template [Common Coordinate Framework version 3 (CCFv3); Wang et al., 2020] using segmentations of the mouse brain atlas. In the case of P56 data, ISH data already aligned to the CCFv3 template by the Allen Institute were directly acquired. To allow for comparisons with the MRI data, ANTs was used to register the CCFv3 template (over which the expression data are defined) to the MRI template (over which the volume changes are defined), thus allowing for comparisons of volume change to gene expression. To determine the association between volume loss and Gad2 expression at E18.5 and P56 (as a reflection of knock-in Cre activity), we computed volume changes for each region using RMINC package for R (Team, 2018) and ran linear models independently for each region, modeling the regional volume as a function of genotype, resulting in a $t$ statistic for each region. Finally, for the E18.5 and P56 separately, we determined the association between Gad2 expression and volume change by using a linear model to predict the $t$ statistic (volume change) for each region from the ISH expression level of that region.

\section{Histology}

Mice were either anesthetized by hypothermia (P5 or younger), with an overdose of sodium pentobarbital ( $>\mathrm{P} 6$ and older) or under isofluorane (4\% in $\mathrm{O}_{2}$ for induction; $1.5-2 \%$ in $\mathrm{O}_{2}$ for maintenance), and transcardially perfused with cold PBS followed by $4 \%$ PFA. Brains were postfixed in $4 \%$ PFA overnight or for $2 \mathrm{~h}$ at $4^{\circ} \mathrm{C}$, and then rinsed and stored in PBS with $0.06 \%$ sodium azide. Postnatal brains were cryopreserved in $30 \%$ sucrose, embedded and frozen in OCT cryopreservation agent (Tissue-Tek), and coronal sections were collected at $20 \mu \mathrm{m}$ using a Cryostat microtome. To prepared floating coronal sections from $>$ P28 animals, brains were cryopreserved in $30 \%$ sucrose and cryosectioned at $35 \mu \mathrm{m}$. Sagittal cerebellar sections were prepared at $75 \mu \mathrm{m}$ using a vibratome, or at $25-35 \mu \mathrm{m}$ using a cryostat.

Slides with postnatal cryosections were incubated successively with blocking solution ( $0.3 \%$ Triton X-100, 5\% normal donkey serum in PBS) and primary antibodies (overnight at $4^{\circ} \mathrm{C}$ ). Floating brain slices were rinsed with $0.5 \%$ PBS-Triton X-100 and incubated in the blocking solution (8\% NDS in PBS) for $1.5 \mathrm{~h}$ at room temperature, then with primary antibodies for $48-72 \mathrm{~h}$ at $4^{\circ} \mathrm{C}$. After $3 \times 10 \mathrm{~min}$ washes in PBS, sections were incubated for $2 \mathrm{~h}$ at room temperature with Alexa Fluor-conjugated secondary antibodies (Thermo Fisher Scientific or Jackson ImmunoResearch). Nuclei were labeled with DAPI, or neuronal somata were stained with NeuroTrace 435/455 Blue Fluorescent Nissl (Thermo Fisher Scientific). Tissue was mounted and cover-slipped using Fluoromount-G (Southern Biotech) or Dako Mounting Medium (Agilent Technologies,).

The primary antibodies used for this study are: goat anti-PV (1:2000, Swant, RRID:AB_2650496); rabbit anti-somatostatin (SST; 1:1000, Peninsula Laboratories, RRID:AB_518614); mouse anti-Reelin (1:1500, Millipore, RRID:AB_2179313), mouse anti-Gad67 (1:1000, Millipore, RRID:AB_2278725), rabbit anti-Vasoactive intestinal peptide (VIP; 1:1000, Immunostar, RRID:AB_572270); rabbit anti-calbindin (1:5000, Swant, RRID:AB_2721225); chicken anti-RFP (1:1500, Millipore, RRID: AB_91496); rabbit anti-RFP/DsRed (1:2000, Rockland, RRID:AB_ 2209751); rabbit anti-cleaved Caspase-3 (1:300, Cell Signaling Technology, RRID:AB_2341188); rabbit anti-cleaved Caspase-9 (1:500, Cell 
Signaling Technology, RRID:AB_2073476); rabbit anti-FoxO3a (1:750, Cell Signaling Technologies, RRID:AB_836876).

\section{Cell counting}

For all quantifications, slides were coded and counts were performed with the examiner blind to the genotype. Immunofluorescence samples were imaged using a Leica SP8 confocal microscope. Confocal images were analyzed as a $\mathrm{z}$-stack or rendered into maximum projections using LasX (Leica; RRID:SCR_013673) or Fiji software (RRID:SCR_002285; Schindelin et al., 2012).

\section{Stereology}

Sections from selected brain regions were imaged [globus pallidus (GP); basolateral amygdala (BLA); somatosensory cortex, S1BF; and primary visual cortex (V1). A section sampling fraction (ssf) for each region was determined, with the first section randomly selected for an unbiased series of sections. After calculating the total area of the region, the optical dissector method was used to select points within the region at $5 \times$ magnification, and observed at a $63 \times$ magnification to obtain optical $z$-stack images from each region. Cell counts were collected from $\mathrm{z}$-stack images at a thickness of $1.8 \mu \mathrm{m}$, for a total of $\sim 32 \mu \mathrm{m}$, and $1024 \times 1024$ pixels. A guard zone of $3.6 \mu \mathrm{m}$ at each superficial portion of the slice was selected to prevent bias because of oversaturation or cutting artefacts. Total PV + cell numbers for each region were estimated using the optical fractionator method (Mayhew and Gundersen, 1996). For the S1BF (bregma +0.38 to $-1.94 \mathrm{~mm}$ ), the $s s f=1 / 6$, the counting frame was set at $49.50 \times 49.50 \mu \mathrm{m}$ and the grid size was $171.17 \times 171.17 \mu \mathrm{m}$. For the GP [bregma $+0.02 \mathrm{~mm}$ to $-1.70 \mathrm{~mm}$ ], the $\mathrm{ssf}=1 / 4$, the counting frame was set at $39.96 \times 39.96 \mu \mathrm{m}$, and the grid size was $237.45 \times 237.45 \mu \mathrm{m}$. For the BLA [bregma -0.58 to $-2.06 \mathrm{~mm}$ ], the $s s f=1 / 6$, the counting frame was set at $49.50 \times 49.50 \mu \mathrm{m}$, and the grid size was $158 \times 158 \mu \mathrm{m}$. For the V1, the $s s f=1 / 7$, the counting frame was set at $43.20 \times 43.20 \mu \mathrm{m}$, and the grid size was $132 \times 132 \mu \mathrm{m}$.

\section{Quantifications of cINs}

Composite images spanning the primary somatosensory cortex (SIBF) were acquired by stitching a grid of 9-12 confocal images with $20 \times 1$ $0.75 \mathrm{NA}$ objective and $1.8 \mu \mathrm{m}$ z-step size. Cell counts for PV,+ SST + , Reelin + , VIP + classes were obtained within a specified region of interest (ROI; width, $1380 \mu \mathrm{m} \times$ height, spanning Layers I-VI) and containing a maximum projection with $18 \mu \mathrm{m}$ z-depth. Cortical layers were determined with DAPI counterstaining and cell counts were subdivided into Layers I-III, IV, and V-VI, using ImageJ/Fiji and the Cell Counter plug-in. Areas of total and layer-specific ROIs were measured using ImageJ. Density was calculated as counts divided by the area of the maximum projection.

For counts of migrating interneurons, coronal cryosections of E14.5 embryonic brains were collected from $P c d h g^{W T}$ or $P c d h g^{H e t}$ and from Pcdhg $g^{f / f}$ Gad2-Cre; Ai14-TdTomato littermates. Confocal images were collected $\left(20 \times\right.$, glycerol, $1.2 \mu \mathrm{m} \mathrm{z}$-step) and an ROI $\left(\sim 50,000 \mu \mathrm{m}^{2}\right)$ spanning the entire cortex in the middle of the tangential migratory stream was analyzed. Manual counts of TdTomato + cells from maximum projection images were completed using ImageJ. Cell counts were divided by the ROI area and reported as cells $/ \mathrm{mm}^{2}$.

For counts of Gad2-Cre; tdTomato-labeled interneurons through development, stitched images of barrel cortex were taken at $40 \times / 1.4 \mathrm{NA}$. Using Imaris (BitPlane), images were segmented by cortical layers and ROIs were drawn based on DAPI staining to delineate cortical plate, Layer V, Layer VI in P2-P5 animals, or Layers II/III, IV, V-VI in P8P14 animals. The ROIs were extended in the $z$-axis to generate a $15-\mu \mathrm{m}$ thick volume. This surface was used to mask the tdTomato channel, followed by spot detection on the tdTomato mask (estimated spot diameter of 8-12 $\mu \mathrm{m}$ ). Detected spots were manually validated to count only those spots with corresponding DAPI and tdTomato signals. For older time points, astrocytes were manually excluded based on morphology. To obtain a cell density value, the number of tdTomato + spots was divided by the total volume of the masking surface for each layer, or summed across layers. A minimum of four ROIs per layer, per animal, and three mutant (Pcdhg f/f; Gad2-Cre; Ai14-TdTomato) and three control (Pcdhg ${ }^{W T}$ or Pcdhg ${ }^{\text {Het }}$; Gad2-Cre; Ail4-TdTomato) genotypes were analyzed. For apoptotic counts, P7 coronal sections spanning the somatosensory to visual cortical regions were stained simultaneously for cleaved Caspase- 3 and Caspase- 9 , and the TdTomato+/CC $3 / 9+$ signals were counted in the entire cortical sections.

\section{Quantification of cerebellar molecular layer interneurons (MLIs)}

For measurements of parvalbumin (PV) + interneuron and calbindin + Purkinje cell number at maturity, $276 \times 276 \mu \mathrm{m}$ ROIs were sampled in folia 5 and $6 . \mathrm{PV}+$ interneurons in the molecular layer were counted in three ROIs per cerebellar sagittal section, and the average was reported per section. Each counting frame consisted of one inclusion and one exclusion edge, and cells were counted if found entirely within the counting frame or overlapping with the inclusion edge but not the exclusion edge. For measurements of TdTomato + MLIs during development, composite images spanning the entire folia 5 were obtained and cells were counted within the molecular layer. Two sections were counted per animal, and two imaging planes were counted per section. The average was reported per animal. For apoptotic MLI counts, combined cleaved Caspase- $3+$ and Caspase-9+ signals overlapping with TdTomato were counted within the molecular across all cerebellar folia of $35-\mu \mathrm{m}$-thick sections. The total area of the molecular layer was measured using the Fiji measurement tool, and Caspase- $3+/ 9+$ cells were divided by the area of the maximum projection to calculate the number of apoptotic cells $/ \mathrm{mm}^{2}$.

\section{Image analysis}

Fluorescence intensity of FoxO3A immunostaining was analyzed using the surface detection algorithm in Imaris (BitPlane, RRID:SCR_007370) to segment the tdTomato + cells of interest in each image, followed by segmentation of DAPI-labeled nuclear surfaces. The FoxO3A+ channel was masked using both of these surfaces to segment cytosolic and nuclear compartments, and the ratio of nuclear to cytoplasmic fluorescence intensity was obtained to report the proportional FoxO3A subcellular localization.

\section{Single molecule fluorescence in situ hybridization (smFISH)}

HCR amplification-based smFISH was performed following previous methods (Choi et al., 2010; Moffitt et al., 2016; Moffitt and Zhuang, 2016). P7 WT cortex was sectioned at $16 \mu \mathrm{m}$ onto $12-\mathrm{mm}$ silane-treated coverslips. Primary probe hybridization targeting the Pcdhg constant region was performed at $2 \mathrm{nM}$ probe concentration in HCR hybridization buffer (Molecular Instruments) at $37^{\circ} \mathrm{C}$ overnight; $4 \%$ polyacrylamide gels were cast for tissue clearing according to previous descriptions (Moffitt et al., 2016; Moffitt and Zhuang, 2016). Gels incubated for $30 \mathrm{~min}$ at $4^{\circ} \mathrm{C}$, before gelation at $37^{\circ} \mathrm{C}$ for $2 \mathrm{~h}$. Tissue clearing occurred overnight at $37^{\circ} \mathrm{C}$ in clearing digestion buffer (Moffitt et al., 2016; Moffitt and Zhuang, 2016). HCR amplification occurred at room temperature for $12 \mathrm{~h}$.

Immunoprecipitation and Western blotting from FACS-sorted cINs TdTomato-expressing cells were isolated by FACS from Pcdhg ${ }^{f}$; Gad2cre;Ai14-tdTomato mice at P7. Cortical cell dissociation was conducted as described for analytical flow cytometry experiments with the following modifications. After dissociation, $1 \mathrm{ml}$ of the cell suspension was layered onto $4 \mathrm{ml}$ of $22 \%$ Percoll (Sigma P1644) + PBS and spun at $400 \times g$, $4^{\circ} \mathrm{C}$, for $10 \mathrm{~min}$ without centrifuge brakes engaged. The supernatant was removed, cells resuspended in $500 \mu \mathrm{l} 2 \%$ HI-FBS + PBS (ThermoFisher), and passed through a $40-\mu \mathrm{m}$ cell strainer. DAPI was added as a viability marker (Thermo Fisher Scientific, RRID:AB 2629482; $5 \mu \mathrm{M})$. Using a Beckman Coulter MoFlo Astrios VBYR cell sorter, an approximately equal number of TdTomato-expressing cells were collected from each genotype. Isolated cells were pelleted $(400 \times \mathrm{g}$, $4^{\circ} \mathrm{C}$ ) and resuspended in $100 \mu$ l lysis buffer (ThermoFisher 87787 ) containing protease (ThermoFisher A32955) and phosphatase inhibitors (ThermoFisher 78 442). Lysis cocktail was incubated on ice in the dark for $20 \mathrm{~min}$ with intermittent vortexing. To remove debris, lysates were spun at $13,000 \times g, 4^{\circ} \mathrm{C}$, for $10 \mathrm{~min}$. Supernatant was aliquoted, flash frozen and stored at $-80^{\circ} \mathrm{C}$. PanAKT was immunoprecipitated from the 
A

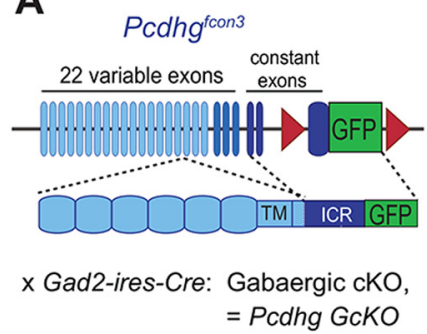

D

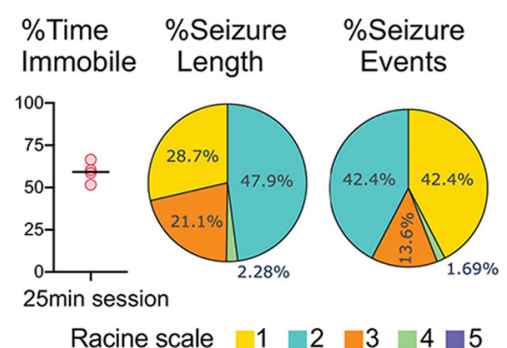

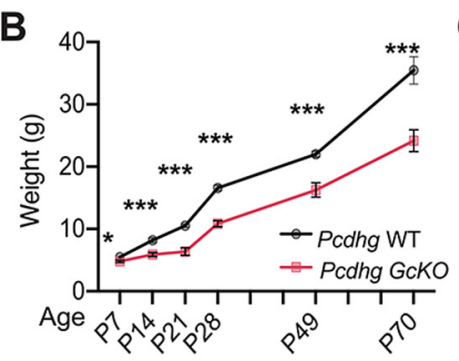

C

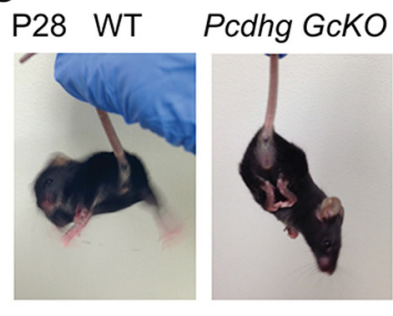

E

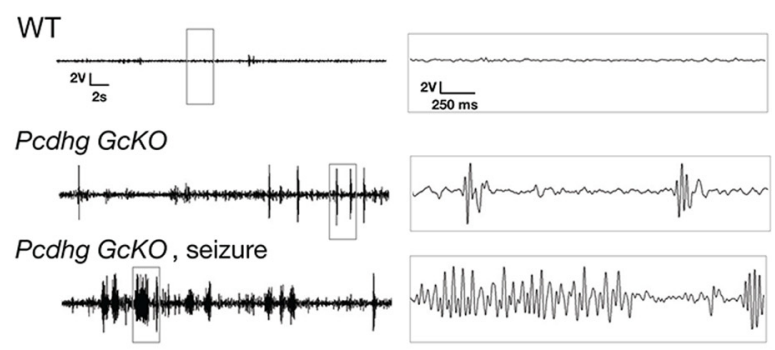

Figure 1. Mice with pan-GABAergic deletion of Pcdhgs exhibit neurologic deficits and seizures. A, Schematic of the LoxP-targeted Pcdhg locus, Pcdhg ${ }^{\text {fcon3 }}$. Distinct PCDHG isoforms are assembled by splicing one of 22 variable exons, encoding the extracellular and transmembrane (TM) regions unique to each isoform, to three constant exons encoding the intracellular constant region (ICR) shared by all 22 PCDHGS. B, Weights of control Pcdhg ${ }^{\text {WT }}$; Gad2-Cre (black) and Pcdhg ${ }^{\text {fff; }}$; Gad2-Cre (herein referred to Pcdhg GcKO, red) mice; Data points show mean \pm SEM ${ }^{*} p<0.05,{ }^{* * *} p<0.001$. C, left, P28 WT sibling. Right, Pcdhg GcKO mutant displays forelimb and hindlimb clasping. D, Total time immobile of four Pcdhg GcKO mice recorded by EEG and video during 25 -min sessions (left, percent time immobile) was analyzed and categorized as seizure events according to the Racine scale, and reported as percent length and events. $\boldsymbol{E}$, Examples of EEG waveforms of cortical baseline activities recorded with bipolar electrodes in WT and $P c d h g$ GcKO mice ( $N=4$ per genotype).

lysate using the rabbit anti-mouse AKT antibody (1:1000; Cell Signaling Technologies, RRID:AB_915783) and the PierceTM Classic Magnetic IP/Co-IP kit (ThermoFisher 88804). The IP product was then run on a Western blotting and probed using both the rabbit anti-mouse panAKT (1:1000) and phospho-AKT (Ser473, 1:1000, Cell Signaling Technologies, RRID:AB_2315049) antibodies, using LI-COR blocking buffer (LIC0927-70 001). FACS-isolated SVZ cells were included as positive controls. IgG raised in rabbit was used as an isotype control (Cell Signaling Technologies, RRID:AB_1031062). To visualize bands, the LICOR IRDye 800CW (LIC-926-32 211) and FC Odyssey imaging system were used. Band size was determined using the PageRuler Plus prestained ladder (LI-COR 26 619).

\section{Analytical flow cytometry}

Population specific flow cytometric analysis of pAKT immunoreactivity in TdTomato-expressing cINs was conducted using Pcdhg; Gad2-cre; Ail4-tdTomato transgenic mice at P7. Brains from two Pcdhg f/f and control heterozygous $P c d h g^{+/ f}$ littermates were included in each experimental replicate. To dissociate cells, neocortical tissue was dissected in ice-cold $\mathrm{Ca}^{2+} / \mathrm{Mg}^{2+}$-free HBSS containing: $10 \mathrm{~mm}$ HEPES, 1 mM Na-pyruvate, and $0.1 \% \mathrm{D}$-glucose. Tissue was cut into $\sim 2-\mathrm{mm}^{2}$ pieces and transferred to 4-ml ice-cold Hibernate A (ThermoFisher, catalog \#A1247501) containing $1 \times \mathrm{B} 27^{\mathrm{TM}}$ supplement. With no prior enzymatic digestion, tissue was triturated using a series of blunt tip stainless steel needles of descending gauge: $25,22,18 \mathrm{~g} 4 \times$ passages each (McMaster-Carr Supply, catalog \#75165A687, \#75165A683, \#75165A676). Cells were filtered through a 70- $\mu$ m nylon mesh cell strainer, pelleted by centrifugation at $400 \times g$, and suspended in 5 $\mathrm{ml}$ ice-cold PBS $+4 \%$ PFA for $20 \mathrm{~min}$. Fixed cells were washed twice in $10 \mathrm{ml}$ PBS, permeabilized in $500-\mu \mathrm{l} 0.3 \%$ Triton X-100 $\mathrm{PBS}+2 \% \mathrm{BSA}$ for $5 \mathrm{~min}$, and incubated for $30 \mathrm{~min}$ in the primary + secondary antibody solution: $2 \%$ BSA-PBS + rabbit antimouse phospho-AKT (Ser473) antibody (1:50, Cell Signaling Technologies RRID:AB_2315049), goat anti-rabbit Alexa Fluor 488 secondary antibody (1:1000 ThermoFisher, RRID:AB 2633280), and PureBlu Hoechst 33342 nuclear dye (Bio-Rad, cata$\log \# 135-1304)$. Cells were washed twice in PBS to remove excess antibody, resuspended in 500- $\mu$ l PBS, and passed through a $40-\mu \mathrm{m}$ nylon mesh cell strainer. Flow cytometry was conducted using a Becton-Dickinson LSRII-CFI, VBGR analyzer and data processed using FlowJo software v10.6.1. Approximately 10,000-15,000 TdTomato+ events were recorded in each flow analysis. Specificity controls included: unlabeled TdTomato + cells and TdTomato + cells $+2^{\circ}$-antibody/Hoechst 33342 .

\section{Statistical analyses}

Statistical analyses were performed in GraphPad Prism. Means of two groups were compared using the two-tailed unpaired $t$ test on condition of equivalent variances and normality or with the Mann-Whitney nonparametric $U$ test. Means of multiple samples were compared using one-way ANOVA and post hoc Tukey's or Holm-Sidak's multiple comparison tests. Other statistical tests are specified in figure legends.

\section{Results}

\section{GABAergic-specific deletion of pcdhgs in mice causes neurologic deficits}

To investigate the role of the PCDHGs in inhibitory neuron development, conditional Pcdhg mice were crossed to the Gad2ires-Cre line to delete the Pcdhgs (Fig. 1A) from broad classes of differentiating GABAergic cells during embryonic development (Taniguchi et al., 2011; Taniguchi, 2014). Homozygous Pcdhgff; Gad2-ires-Cre mutant offspring (herein referred to as Pcdhg $G c K O)$ were viable but displayed pronounced growth and neurologic deficits within the first postnatal weeks. By P28, Pcdhg GcKO mutants were on average $30 \%$ smaller in body weight than wild-type littermates (16.6 g compared with $10.8 \mathrm{~g}, N=15$ animals per genotype, $p=0.00009$, unpaired $t$ test; Fig. $1 B$ ). Juvenile Pcdhg GcKO mice exhibited forelimb and hindlimb clasping on tail suspension, a neurologic phenotype that occurred with $100 \%$ penetrance (Fig. 1C).

We observed that Pcdhg GcKO mutant mice exhibited spontaneous, convulsive seizures. Cortical EEG recordings were combined with video to monitor wild-type sibling and Pcdhg GcKO 
A

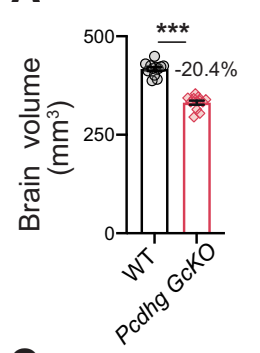

C Globus Pallidus
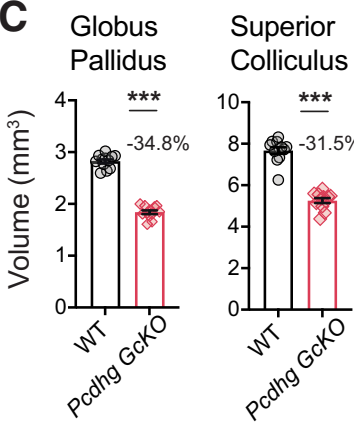

B

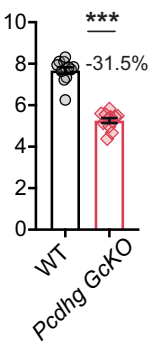

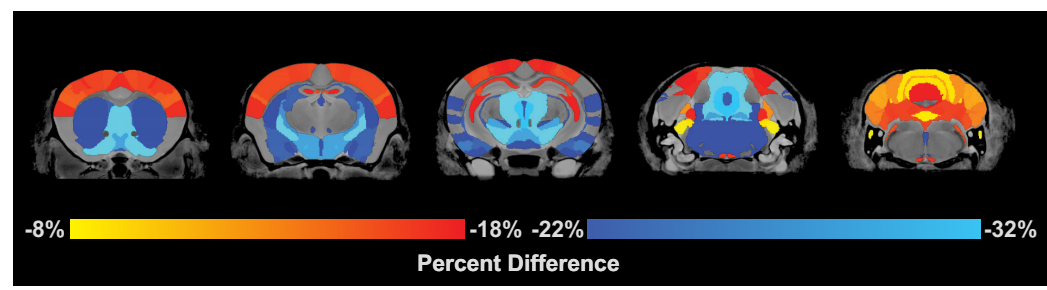

Percent Difference

E

Amygdala

Somatosensory
Cortex S1BF

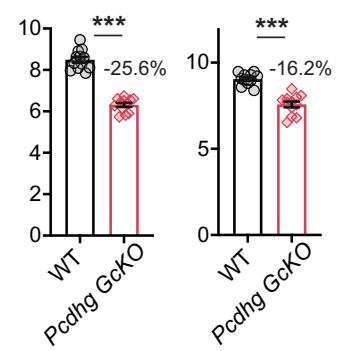

D
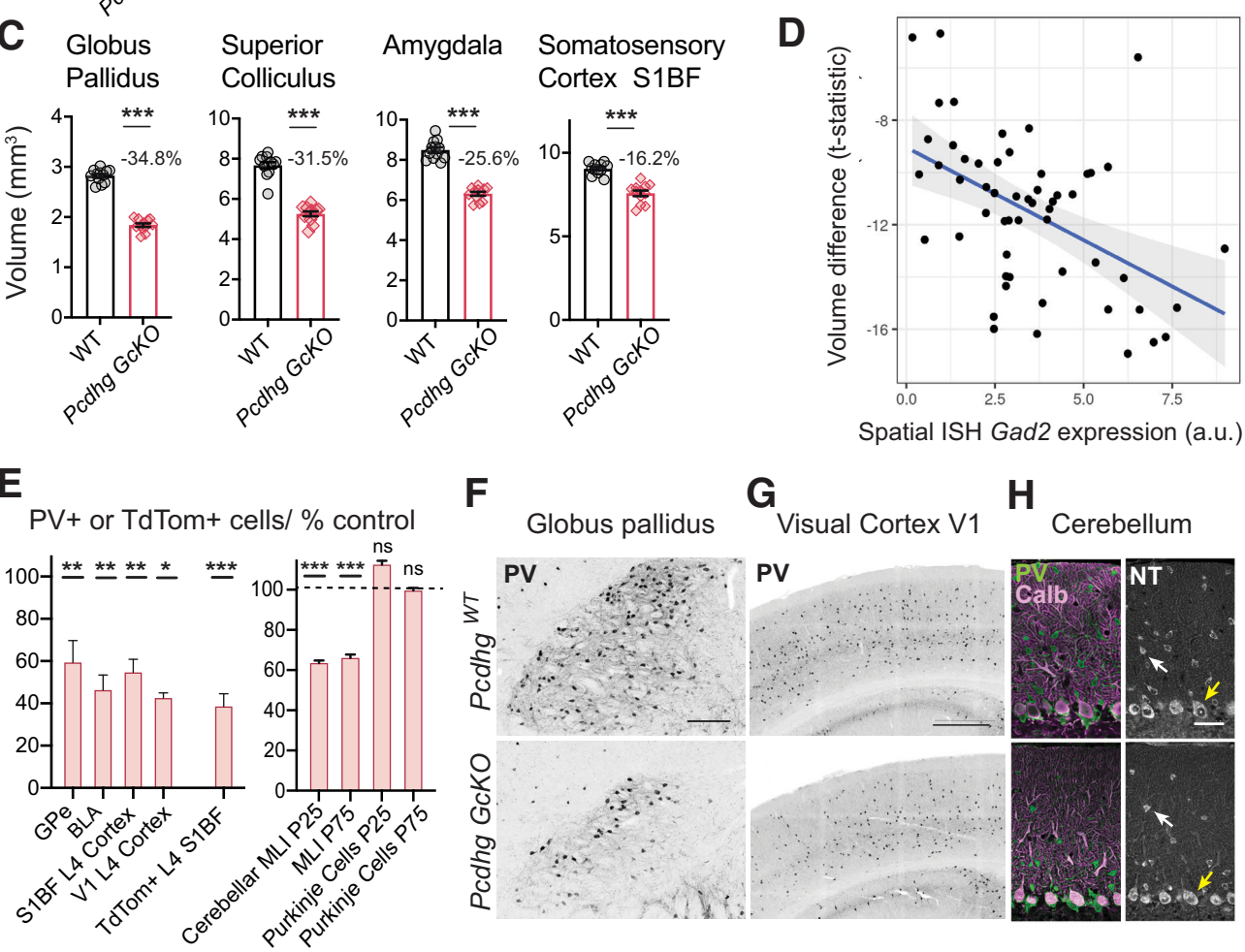

F

Globus pallidus

G
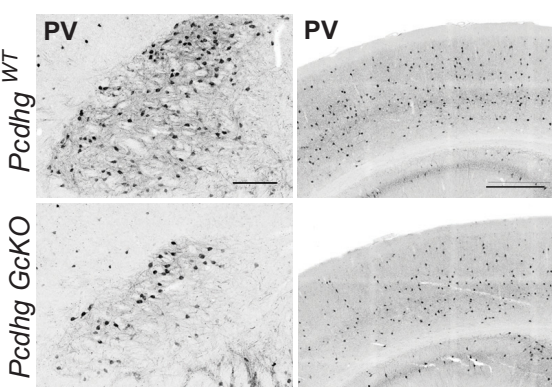

$\mathrm{H}$

Cerebellum

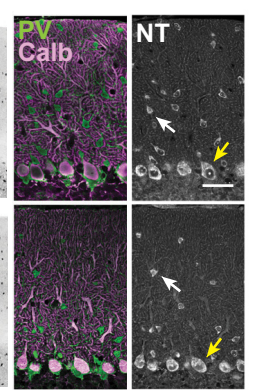

Figure 2. Pcdhg GCKO mutants have reduced brain volumes and PV-expressing populations. A, MRI quantification of absolute brain volumes of Pcdhg ${ }^{\text {WT }}$ (control, black) and Pcdhg GcKO (red) mutants at P28. Data show mean volume \pm SEM from 12 animals per genotype; $p<0.00001$, unpaired Student's $t$ test. $\boldsymbol{B}$, Coronal examples of whole-brain MRI data highlight regional reductions in Pcdhg GcKO brains, expressed as percent reduction from control brains. Regions coded in blue are significantly reduced in volume compared with the mean whole-brain reduction $(-20.4 \%)$ and those coded in red-yellow are less severely affected, with significance exceeding FDR threshold at $p=0.05$. C, Volumes of selected regions of control (black) and Pcdhg GcKO (red) brains. $\boldsymbol{D}$, MRI volume changes are negatively correlated with spatial E18.5 Gad2 mRNA expression obtained from the Allen Brain Institute Developing Mouse Brain ISH dataset $(t$ statistic $=$ $-4.10, p=0.000144)$. Each point corresponds to a segmented brain structure, and plotted for volume changes computed from MRI measurements and spatial Gad2 gene expression. $E$, left, Quantifications of PV-expressing (PV + ) cells in the Pcdhg GCKO GP $(p=0.0087)$, BLA $(p=0.0042)$, somatosensory cortex SIBF Layer IV ( $p=0.011)$, V1 Layer IV $(p=0.016)$, and Gad2-Cre; Ai14TdTomato-labeled neurons in SIBF Layer IV ( $p=0.00002$; all three to four animals per genotype). Right, Quantifications of PV + MLLs $(p<0.00001)$ and Purkinje cells (PCs; $p=0.08)$ at P25 (five to seven animals per genotype) and at P75 (three animals per genotype). Bars show means from Pcdhg GCKO mice normalized as percentage from control counts, with \% SEM, unpaired $t$ test. $F$, G, Inverted immunofluorescence images of PV-expressing (PV+) cells in GP (F) and visual cortex (G) in control and Pcdhg GcKO mice. $\boldsymbol{H}$, left, Immunostaining of PV+ MLIs (green) and PV+/calbindin + PCs in cerebellar cortex. Right, Soma labeling by NeuroTrace. MLI density (white arrow) is reduced but PC density is unchanged (yellow arrow) in Pcdhg GcKO cerebella. ${ }^{*} p<0.05,{ }^{* *} p<0.01$, ${ }^{* * *} p<0.001$. Scale bars: $500 \mu \mathrm{m}(\boldsymbol{F}), 200 \mu \mathrm{m}(\boldsymbol{G})$, and $25 \mu \mathrm{m}(\boldsymbol{H})$.

mutant mice (three months of age, $N=4$ animals per genotype). Periods of extended immobility were identified and assessed for behavioral seizures using a modified Racine scale (Racine, 1972; Kim et al., 2019). During these periods (immobile $59 \pm 3 \%$ SEM of recorded time; $N=4$ animals), $P c d h g$ GcKO mutant exhibited seizure symptoms and convulsive behaviors, including stiff posturing (scale 1), nodding and forelimb clonus (scale 2, 3), and in a few severe instances, continuous whole-body seizures (scale 4; Fig. 1D). Analysis of simultaneous EEG recordings of baseline cortical activities revealed abnormal bursting and epileptiform discharges during seizure episodes in all mutant Pcdhg GcKO animals but not in wild type (Fig. $1 E$ ). The neurologic impairments exhibited by Pcdhg GcKO mutants indicate severe defects in the development or function of the GABAergic circuitry in the brain.

\section{GABAergic Pcdhg mutants display reductions in brain} volume and GABAergic populations

Juvenile Pcdhg GcKO mutants developed smaller brains. To locate the anatomic phenotypes, Pcdhg GcKO brains were analyzed ex vivo by MRI. The total brain volumes of Pcdhg GcKO mutants were reduced by $20.5 \%$ compared with $P c d h g^{\mathrm{WT}}$; Gad2Cre control littermates $(N=12$ animals per genotype; $p<$ 0.00001 , unpaired $t$ test; Fig. $2 A$ ). To identify structure-specific differences, the brains were segmented into 182 structures and measured as percent volume differences from control. Although Pcdhg GcKO mice are smaller, region-specific reductions were detected across the brain, indicating that volume changes vary independently from body size (Fig. $2 B$ ). The most profoundly affected structures include the GP, the bed nucleus of stria terminalis and the superior colliculus (Fig. 2C), regions that contain 
high proportions of GABAergic interneuron and projection neurons (Kudo et al., 2012; Saunders et al., 2016; Villalobos et al., 2018; Whyland et al., 2020). Volume changes were less severe in neocortical areas and the amygdala, which proportionally contain fewer GABAergic neurons (Ehrlich et al., 2009; Sahara et al., 2012; Fig. 2C). To test the association between structure-specific reductions and GABAergic neuron content, we projected onto the MRI volume measurements the spatial patterns of Gad2 mRNA expression using the Allen Institute's Mouse Brain in situ hybridization atlases (E18.5 and P56; Lein et al., 2007; Sunkin et al., 2013). Structure-specific volume changes in Pcdhg GcKO brains negatively correlated with spatial Gad2 expression from the E18.5 ( $t$ statistic $=-4.10, p=0.00014$; Fig. $2 D$ ) and P56 atlases $(t=-3.55, p=0.00048$, linear model). Therefore, structures with higher Gad2-positive spatial content, and by extension Cre activity, are associated with greater volume reductions in Pcdhg GcKO mutants.

To determine whether the anatomic changes result from GABAergic neuron loss, we quantified cells marked by PV Parvalbumin (PV) in select regions. We began with the GP externus, where PV + cells comprise $\sim 55 \%$ of the total population and are primarily GABAergic projection neurons that target cortical and subcortical structures (Hernández et al., 2015). In the Pcdhg GcKO GP, PV+ neurons are reduced by $40.7 \%$ compared with $P c d h g^{W T}$ littermates $(N=4$ animals per genotype, $p=0.0087$, unpaired $t$ test; Fig. $2 E, F)$. Interneuronal PV+ populations are also reduced in Pcdhg GcKO mutants, including those in the BLA ( $-53.7 \%, p=0.0042$, unpaired $t$ test), and in the primary visual and somatosensory barrel cortex $(-57.5 \%$, $p=0.0042$ and $-45.4 \%, p=0.0031$, respectively; unpaired $t$ test; Fig. 2E,G). Pcdhg deletion leads to loss of cells rather than loss of PV expression, because interneurons labeled by the Ai14TdTomato Cre reporter in Layer IV of the somatosensory cortex are reduced by $61.5 \%$ in Pcdhg GcKO mutants compared with control ( $p=0.005$, unpaired $t$ test, three animals per genotype; Fig. 2E). Similarly, in the Pcdhg GcKO cerebellar cortex, numbers of PV + MLIs were reduced at P25 $(-36.5 \%, p<0.00001$, unpaired $t$ test; five to seven animals per genotype) and did not decline further. PV+ Purkinje cells, which are GABAergic projection neurons, did not differ in Pcdhg GcKO (Fig. 2E,H), consistent with our previous finding that Purkinje-specific Pcdhg deletion does not affect Purkinje cell number (Lefebvre et al., 2012). Altogether, these results demonstrate a cell type-specific role for the PCDHGs in mediating PV + GABAergic population size in multiple brain regions.

\section{The PCDHGs are required for cortical GABAergic interneuron survival during developmentally-regulated cell death}

The interneuron loss we observed across hindbrain, midbrain, and forebrain structures of $P c d h g$ GcKO brains raises interesting parallels to Bax-dependent interneuron cell death in early postnatal Pcdhg mutant retina and spinal cord (Lefebvre et al., 2008; Prasad et al., 2008). To test for a role in regulating GABAergic interneuron survival in the brain, we focused on the cortical inhibitory interneurons (cINs) which comprise developmentally and molecularly well-characterized subpopulations. During the first postnatal week, $20-40 \%$ of cINs belonging to the cardinal classes deriving from the medial ganglionic eminence (MGE) and caudal ganglionic eminence (CGE) are eliminated by PCD (Southwell et al., 2012; Denaxa et al., 2018; Priya et al., 2018; Wong et al., 2018). Building on the observation of PV + cIN loss in Pcdhg GcKO (Fig. 2E), we tested whether the Pcdhgs are required in broad or specific classes of cINs in the somatosensory cortex. We quantified the two cardinal MGE-derived classes that express PV or Somatostatin (SST+), and the two CGE-derived classes that are defined by VIP + or by Reelin+/SST- expression in Layer I (Fig. 3A; Rudy et al., 2011). The densities of these four classes were significantly decreased in Pcdhg GcKO compared with $P c d h g^{W T}$ controls (Reelin+/SST- LI-III $p=0.0073$, MannWhitney; Vasoactive intestinal peptide LII-III, IV, V-VI counts $F_{(1,12)}=8.5, p=0.013$, two-way ANOVA; PV+ LII-III, IV, V-VI counts, $F_{(1,12)}=49.2, p=0.000014$, two-way ANOVA; SST + LII-III, IV, V-VI counts $F_{(1,12)}=316.5, p<0.000001$, two-way ANOVA; $N=4$ sections per animal, three animals per genotype; Fig. $3 B-F)$. There was no accumulation of cINs in particular cortical layers relative to others, indicating that laminar migration and type-specific distributions are intact in Pcdhg GcKO mutants ( $\chi^{2}$ of layer distribution: VIP $+p=0.69$; $\mathrm{PV}+p=0.84$; $\mathrm{SST}+p=0.84$; Fig. 3D-F). Moreover, we did not detect dose-dependent differences in cIN densities between wild-type and heterozygous animals (PV+: Pcdhg ${ }^{+/+}$; Gad2-Cre $143.8 \pm 5.7$ vs Pcdhg+/-; Gad2-Cre $144.2 \pm 10.4$ cells $/ \mathrm{mm}^{2}, p=0.97$; SST + : Pcdhg ${ }^{\text {WT }} 108.6 \pm 4.2$ vs $P c d h g^{\text {Het }} 103.1 \pm 4.6$ cells $/ \mathrm{mm}^{2}, p=0.40$; Reelin+: Pcdhg ${ }^{\mathrm{WT}} 96.0 \pm 2.9$ vs $P c d h g^{\text {Het }} 92.13 \pm 1.6$ cells $/ \mathrm{mm}^{2}$, $p=0.30$; four to seven animals, unpaired $t$ test with Welch's correction).

Next, we disentangled other potential developmental roles for the Pcdhgs that might affect cIN number. Gad2-Cre recombination activity is detected in the embryonic ganglionic eminence, including in the subventricular and mantle zones known to contain postmitotic immature Gad2-expressing neurons (Stühmer et al., 2002; Chen et al., 2017b), and along interneuron migratory paths (E14.5; Fig. 4A). The density of migrating cINs labeled by TdTomato in Pcdhg GcKO embryos was similar to control littermates at E14.5 (Pcdhg ${ }^{\mathrm{WT}}$ or Het $1208 \pm 53.2$; Pcdhg GcKO $1220 \pm 45.0 \mathrm{TdTom}+$ cells $/ \mathrm{mm}^{2}, p=0.87$, unpaired $t$ test, three animals per group; Fig. $4 A$ ). We also tested whether cIN loss is associated with functional deficits in intrinsic excitability by performing whole-cell current-clamp recordings of Layer $\mathrm{V}$ cINs at P13-P15. Compared with control, the aggregate of Pcdhg GcKO cINs did not differ in Vrest, Rin, and spike number to incremental current injections (Fig. 4B). Therefore, the loss of cINs in Pcdhg GcKO mutants does not result from migration or gross intrinsic excitability defects.

We performed a series of tests to determine whether the PCDHGs specifically regulate cIN survival during the postnatal wave of PCD. First, we confirmed that Pcdhg mRNA is expressed by GABAergic marker Gad1 mRNA-positive cINs in postnatal cortex at P7, corresponding to the peak of cIN PCD (Fig. 5A). To test whether cIN loss in Pcdhg GcKO mutants results from accentuated PCD, we quantified cINs in $P c d h g^{\text {Het }}$ control and Pcdhg GcKO; Gad2-Cre; Ai14-TdTomato cortices from P2 through P14 ( $N=3$ animals per genotype). In both control and Pcdhg GcKO mutants, densities of TdTomato-labeled cINs declined along a time course consistent with cIN PCD (Southwell et al., 2012; Wong et al., 2018; Fig. 5B-D). cIN densities in Pcdhg GcKO mutants were initially comparable to controls at P5 $(84,315 \pm$ 2418 in control vs 88,315 \pm 2418 in $P c d h g^{G c K O}, p=0.66$, MannWhitney) but declined to a greater extent in Pcdhg GcKO mutants by $\mathrm{P} 8$ (reduced by $27.1 \%$ in Pcdhg GcKO compared with controls; $66,304 \pm 2136$ vs $48,320 \pm 1683$ cells $/ \mathrm{mm}^{3}, p=$ 0.000005, Mann-Whitney; Fig. 5B,D). At P2, we observed a modest but statistically significant increase in cIN density in Pcdhg GcKO mutants (13.8\% increase compared with P2 controls, $p=0.028$; Mann-Whitney; Fig. $5 B$ ), which might reflect a 
A

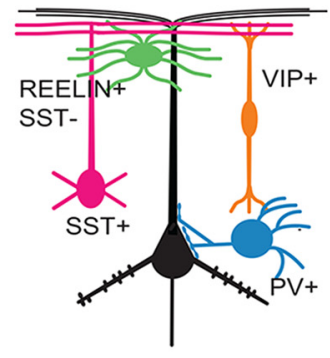

B Pcdhg wT

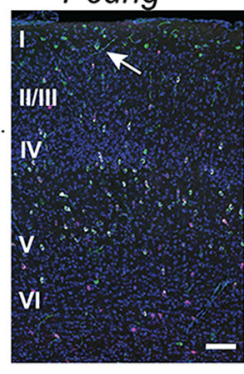

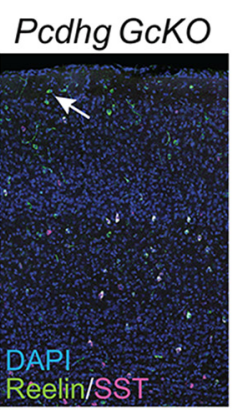

E
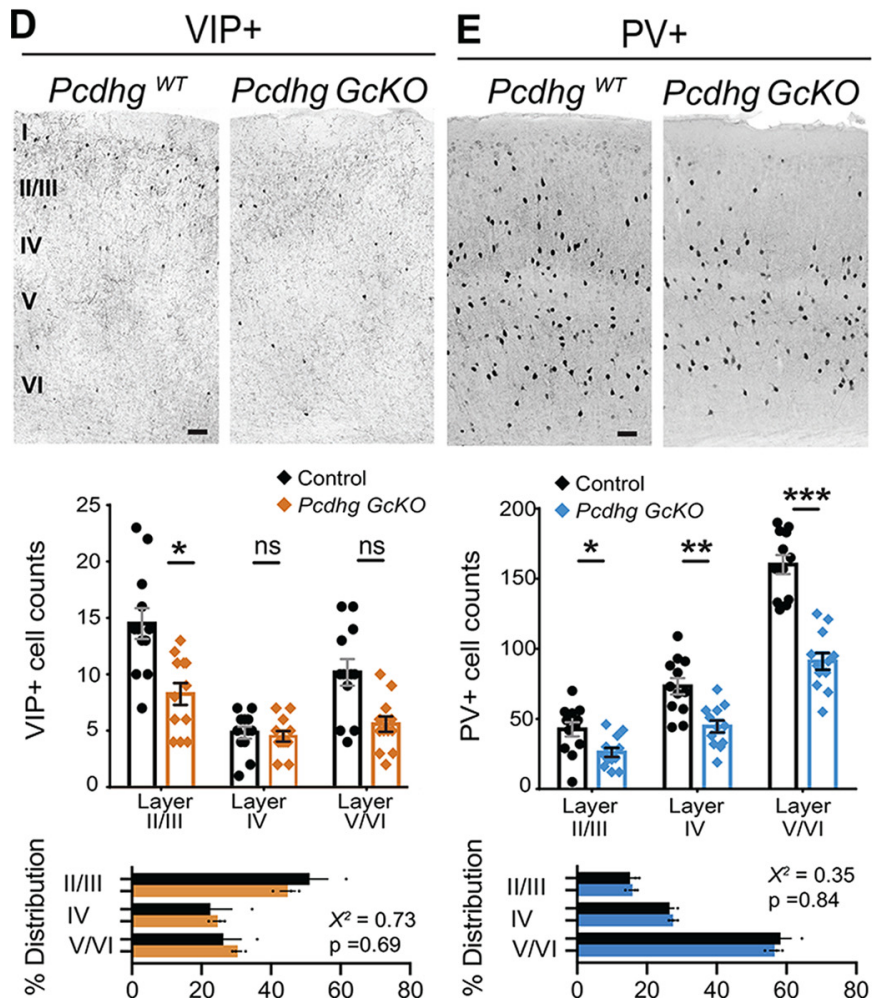

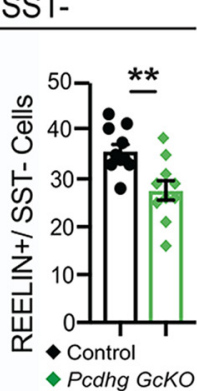

F SST+
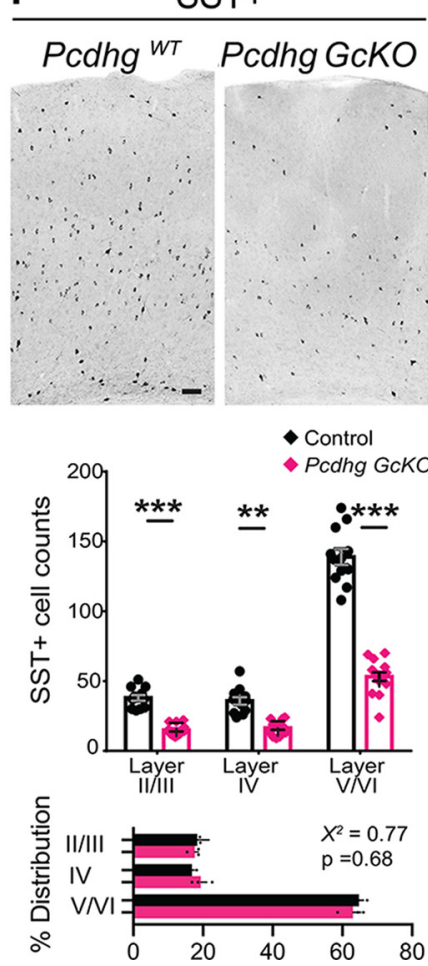

Figure 3. Cardinal classes of cortical GABAergic interneurons are reduced in Pcdhg GcKO mutants. $A$, Schematic depicting cortical pyramidal neuron (black) and markers used to distinguish four interneuron (cIN) types. B-F, Coronal sections through somatosensory cortex (SSC; SIBF) and quantifications of cIN types at P28 of Pcdhg ${ }^{\text {WT }}$ and Pcdhg GcKO animals. Scatter bar plots summarize data, mean \pm SEM from 12 sections, three animals per genotype. $\boldsymbol{B}$, Immunostaining for Reelin (green) and SST (magenta) marks Reelin +/SST- population residing in Layer I (white arrow). C, Labeling and quantification of Reelin +/SST- cINs in defined area from Layers I-III; $p=0.0073$ Mann-Whitney (MW) $U$ test. $\boldsymbol{D}$, Inverted immunofluorescence image (top), quantifications of immunolabeled cells (middle), and layer-specific distribution as a percentage of the total population (bottom). VIP-expressing cINs. Layers II-III: $p=0.016$; IV: $p=0.99 ;$ V-VI: $p=0.30$, two-way ANOVA with Sidak's multiple comparisons. $\chi^{2}$ of layer distribution, $p=0.69$. $\boldsymbol{E}$, PV-expressing cINs. Layers II-III: $p=0.028 ;$ IV: $p=0.0029 ;$ V-VI: $p=0.000026$, two-way ANOVA with Sidak's multiple comparisons. $\chi^{2}, p=0.84$. F, SST-expressing cINs. Layers II-III: $p=0.00025$; IV: $p=0.0017$; V-VI: $p<0.00001$, two-way ANOVA with Sidak's multiple comparisons. $\chi^{2}, p=0.68 ;{ }^{*} p<0.05,{ }^{* *} p<0.01,{ }^{* * *} p<0.001$. Scale bars: $100 \mu \mathrm{m}$.

trend in reduced cortical volumes $(11.9 \%$ reduction; $0.0052 \pm$ $0.00017 \mathrm{~mm}^{3}$ mean ROI volumes for controls vs $0.0046 \pm$ $0.00029 \mathrm{~mm}^{3}$ ROIs for Pcdhg GcKO, $p=0.08$, unpaired $t$ test). Quantification of apoptotic TdTomato + cINs simultaneously marked by cleaved Caspase- 3 and Caspase- 9 confirmed a complementary increase in apoptosis in Pcdhg GcKO cortex at P7 (Fig. 5E; control, $18 \pm 1.0$; Pcdhg GcKO, $24 \pm 1.5 \mathrm{CC} 3 / 9+$ cells, $p=0.009$, Mann-Whitney). Thus, the excess postnatal loss of cINs in Pcdhg GcKO mutants is associated with accentuated cell death.

To test whether the pro-survival requirement for the PCDHGs is temporally restricted to PCD, Pcdhgs were deleted from cINs using PV-Cre, which has an onset of expression in maturing PV + cINs after the first postnatal week (Lazarus et al., 2015). In this experiment in which the Pcdhg genes remain intact during peak PCD, the PV + cell densities in the cortex of Pcdhgff;
PV-Cre animals were indistinguishable from control $\left(P c d h g^{f /+}\right.$ $160.6 \pm 5.1$ vs $P c d h g^{f / f} 169.9 \pm 10.1$ cells $/ \mathrm{mm}^{2}$, three animals per genotype, $p=0.46$, unpaired $t$ test; Fig. $5 F$ ).

In a final test to show that regulation of cIN number by the PCDHGs is specific to PCD rather than other degenerative processes, we tested whether cIN loss in Pcdhg GcKO mutants occurs through the BAX-dependent apoptotic pathway. In constitutive or conditional Bax mutant mice, genetic blockade of apoptosis prevents the natural elimination of cINs (Southwell et al., 2012; Priya et al., 2018; Wong et al., 2018). If excess cIN loss in Pcdhg $G c K O$ cortex results from the overactivation of apoptosis, then the cINs destined to die in Pcdhg GcKO; Bax double mutants (Pcdhgff; Bax flf; Gad2-Cre) would be preserved and result in greater numbers of mature cINs. Indeed, the PV + and Reelin+ cIN populations in Pcdhg GcKO; Bax GcKO double mutant animals were not only restored compared with Pcdhg GcKO single 

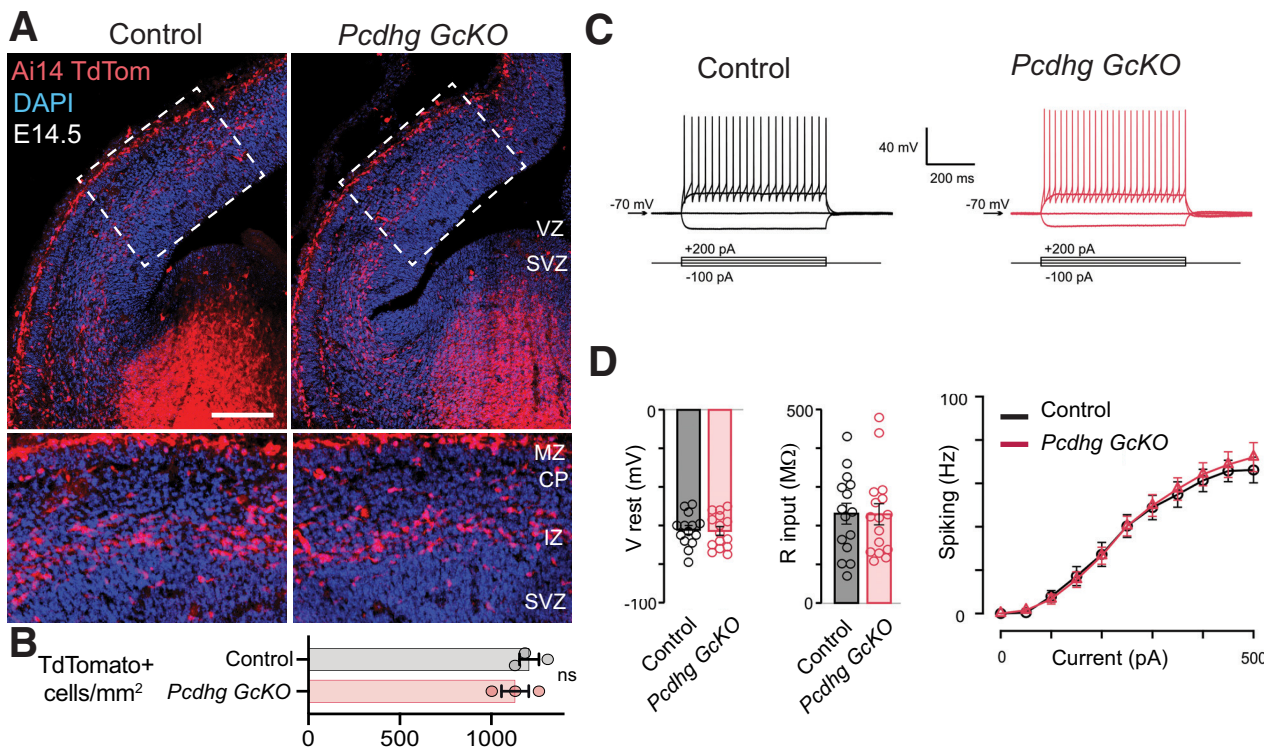

Figure 4. Deletion of the Pcdhgs does not affect cIN migration or intrinsic membrane properties. A, Coronal sections of E14.5 embryonic Pcdhg ${ }^{\text {WT or Het }}$ and Pcdhg ${ }^{\text {fff }}$ Gad2-Cre; Ai14-TdTomato brains. Top, TdTomato + Cre reporter labeling (red) confirms Gad2-Cre recombination in the subventricular and mantle zones in the ganglionic eminence, presumptive striatum, and along the interneuron migratory stream. Bottom, Insets from top panels show migratory streams of cINs. MZ, marginal zone. CP, cortical plate. IZ, intermediate zone. SVZ, subventrical zone. $\boldsymbol{B}$, Quantifications of TdTomato + migratory interneurons in boxed ROls, as shown in $\boldsymbol{A}$. Scatter bar plots summarize data from three animals per genotype, mean \pm SEM $p=0.67$, unpaired $t$ test. C, Representative traces show the voltage responses of TdTomato + Layer V interneurons in the visual cortex (cIN) in Pcdhg ${ }^{\text {WT or Het }}$ and Pcdhg GcK0; Ai14-TdTomato mice (upper traces) following current injection (lower traces). All experiments were performed in slices prepared from P13-P15 mice and in the presence of AMPA-receptor, NMDA-receptor, and GABAa-receptor blockers (AP-5, $50 \mu \mathrm{m}, \mathrm{NBQX}, 10 \mu \mathrm{m}$, picrotoxin, $100 \mu \mathrm{m}$, respectively). D, Summary plots of the Vrest (left panel, Vrest: control, $n=14$ cells/8 animals; $P$ cdhg GcKO, $n=15$ cells/10 animals; $p=0.8412, \mathrm{df}=27$, unpaired $t$ test); Rin (middle panel, Rin: control, $n=15$ cells/8 animals; Pcdhg GcKO, $n=16$ cells/10 animals; $p=0.9709$, df $=29$, unpaired $t$ test,); and action potential firing frequencies, showing no significant differences between the control and Pcdhg GcKO mice (right panel, AP firing: control, $n=15$ cells/8 animals; $P$ cdhg GcKO, $n=16$ cells/10 animals; $+50-500 \mathrm{pA}$ current steps, $p=0.7405-0.8609$; Mann-Whitney test). Data summarize mean \pm SEM. Scale bar: $200 \mu \mathrm{m}$.

mutants but were also indistinguishable from control Pcdhg ${ }^{\mathrm{Het}}$; Bax GcKO siblings (PV+: $59.5 \pm 2.4$ cells $/ \mathrm{mm}^{2}$ in Pcdhg GcKO vs $119.7 \pm 8.7$ in Pcdhg GcKO; Bax GcKO vs $126.1 \pm 8.8$ in Pcdhg ${ }^{+}$; Bax GcKO, $p=0.00004$ and $p=0.52$, respectively; Reelin + across all layers: $104.0 \pm 4.8$ cells $/ \mathrm{mm}^{2}$ in $P c d h g$ GcKO vs $181.4 \pm 9.0$ in Pcdhg GcKO; Bax GcKO vs $174.6 \pm 8.7$ in $\mathrm{Pcdhg}^{+}$; Bax GcKO, $p=0.00002$ and $p=0.47$, respectively; CGE Reelin+/SST - in LI-III: $47.9 \pm 4.4$ cells/ $\mathrm{mm}^{2}$ in Pcdhg GcKO vs $104.9 \pm 6.6$ in Pcdhg GcKO; Bax GcKO vs $101.6 \pm 7.3$ in $\mathrm{Pcdhg}^{+}$; Bax GcKO, $p=0.00007$ and $p=0.67$, respectively; Holm-Sidak's multiple comparisons, three to four animals per genotype; Fig. $6 A, B)$. Together, these multiple lines of evidence establish an essential role for the PCDHGs to promote cIN survival during the postnatal period of PCD.

\section{Pro-survival phospho-AKT levels are diminished in pcdhg mutant GABAergic interneurons during PCD}

Whether developing neurons survive or die is determined by the balance of intracellular signals that suppress or initiate the apoptotic cascade (Pfisterer and Khodosevich, 2017; Hollville et al., 2019). The intracellular signals activated downstream of PCDHGs that promote survival of rodent neurons remain unknown. We focused on the serine-threonine kinase AKT pathway, which was recently implicated in cIN PCD. Phospho-AKT (Ser473) levels transiently increase during the peak of cIN death, and conditional deletion of PTEN, an AKT pathway antagonist, results in increased cIN density (Wong et al., 2018). Differences in phospho-AKT levels between control Pcdhg ${ }^{\text {Het }}$ and Pcdhg GcKO cortical lysates could not be distinguished by standard Western blottings. To discriminate cIN-specific changes, Western blottings were performed on dissociated TdTomato-labeled
cINs isolated by FACS. In cIN-enriched P7 lysates, phospho-AKT levels were detectably lower in Pcdhg GcKO samples (Fig. $7 A, B$ ) but quantifications were hampered by the low amounts of material obtained. We used quantitative flow cytometry to measure phospho-AKT levels in dissociated cINs labeled by Gad2-Cre; Ai14-TdTomato (Fig. 7A,C). Across similar numbers of mutant and control TdTomato + cellular events $(\sim 10000)$, the phospho-AKT fluorescence detected among Pcdhg GcKO cINs was significantly reduced compared with the control cIN population (mean \% pAKT +/TdTomato + events among Pcdhg GcKO cINs = $65.3 \pm 1.4 \%$ compared with control, $95.4 \pm 3.2 \%$; six brains per genotype, $p=0.0008$, unpaired $t$ test; Fig. $7 C$ ).

One mechanism by which AKT is anti-apoptotic is through phosphorylation and cytoplasmic retention of the FoxO transcription factors, and prevention of FoxO-mediated transcription of targets such as pro-apoptotic BH3 members Puma and Bim (Gilley et al., 2003; Ambacher et al., 2012). We predicted that diminished AKT signaling in Pcdhg GcKO cINs would result in increased FoxO3A translocation to the nucleus (Fig. 7D). We compared FoxO3A activity during PCD by quantifying the ratios of nuclear (active) and cytoplasmic (inactive) FoxO3A accumulation by immunofluorescence. At P7-P8, FoxO3A was noticeably enriched in the cytoplasm of TdTomato-labeled cINs in control. In the Pcdhg GcKO cortex, FoxO3A signal is higher in the nucleus, as confirmed by the increased nuclear-to-cytoplasmic ratio of FoxO3A + fluorescence $[p<0.00001$, Kolmogorov-Smirnov (KS) test; Fig. $7 E, F]$. The altered activity states of AKT pathway components in Pcdhg mutant cINs provide new candidates to link the PCDHGs and intracellular signaling cascades that promote neuronal survival. 

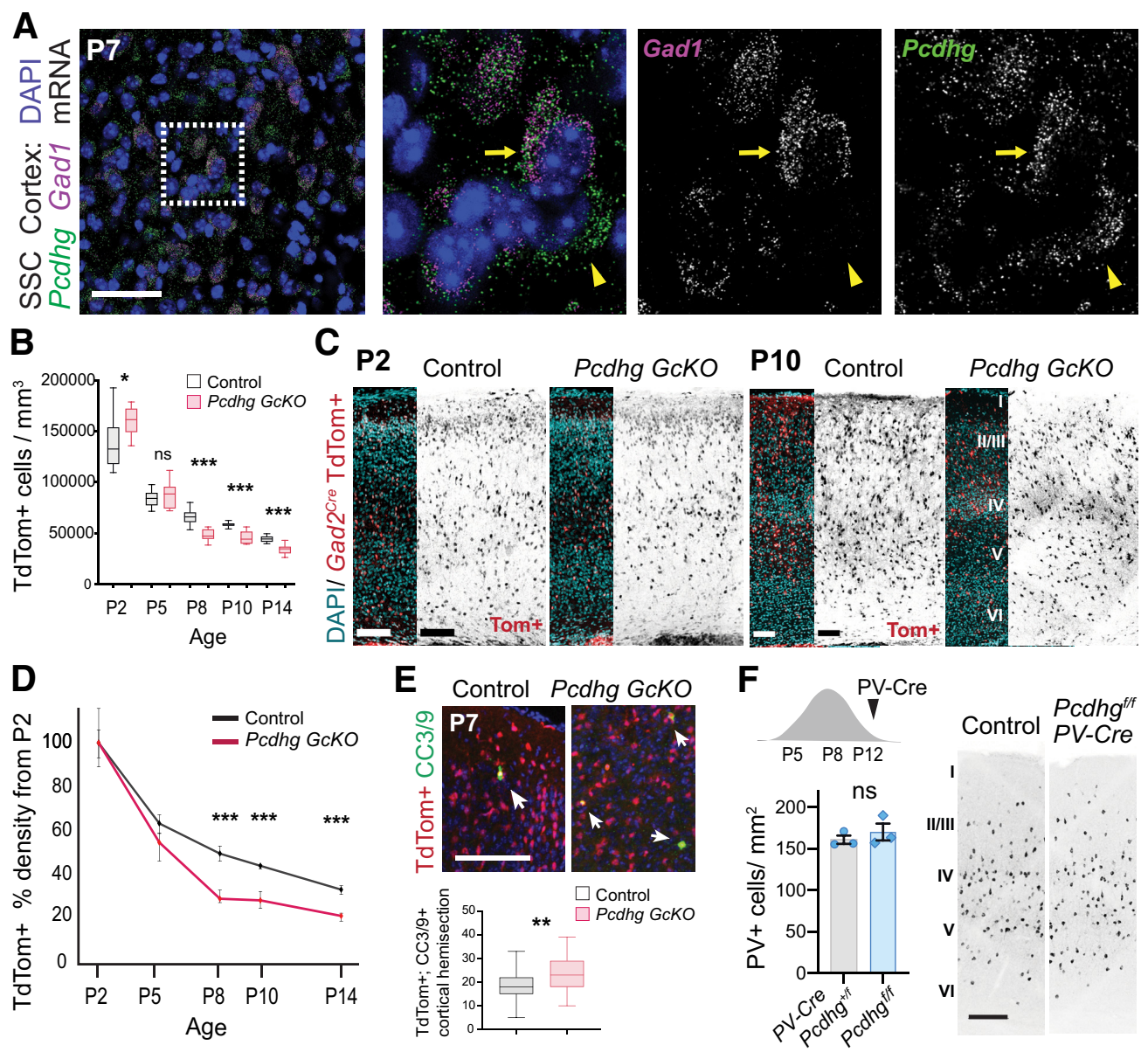

Figure 5. cIN loss in Pcdhg GcKO mutants is the result of accentuated developmental cell death. $\boldsymbol{A}$, FISH of Gad1 mRNA (magenta) and Pcdhg mRNA encoding constant exons common to all Pcdhg isoforms (green) in P7 SSC cortex. Right, Inset shows Pcdhg expression in Gad1 + (arrow) and in Gad1- cells (arrowhead). B, Quantification of cIN density in somatosensory cortex (SSC) through development in Pcdhg ${ }^{\text {WT }}$ or Het control (gray) and Pcdhg GcKO (red). cINs are marked by Gad2-Cre; Ai14-TdTomato. Box plots (with minimum and maximum values) show data from 12 sections, three animals per genotype. P2, $p=0.028 ; \mathrm{P} 5, p=0.66 ; \mathrm{P} 8, p<0.00001 ; \mathrm{P} 10, p=0.0003 ; \mathrm{P} 14, p<0.00001$, Mann-Whitney $U$ test. C, Coronal section of control and Pcdhg GcK0 SSC at P2 and P10 with labeling of nuclei (DAPI, cyan) and TdTomato + cINs (red). Inverted fluorescent images of TdTomato + cINs are on right. D, Graphs show data of TdTomato + cIN densities from $\boldsymbol{B}$, normalized to densities at P2 for control (black) and Pcdhg GcKO (red). P2, $p>0.99 ; \mathrm{P} 5, p=0.096 ; \mathrm{P} 8, p=0.001 ; \mathrm{P} 10, p=0.0027 ; \mathrm{P} 14, p=0.00097$, Mann-Whitney $U$ test. $\boldsymbol{E}$, top, Apoptotic TdTomato + cINs (red) co-labeled with cleaved Caspase-3 and Caspase-9 (CC $3 / 9$ green; white arrows). Bottom, Quantifications of TdTomato/CC $3 / 9+$ cells in P7 cortical sections. Box plots show data from 31 sections, three animals per genotype; $p=0.00095$, Mann-Whitney $U$ test. $\boldsymbol{F}$, top left, Onset of PV-Cre activity occurs after the peak of cIN PCD. Bottom left, PV+ densities in SSC at P28 are indistinguishable between control (gray bars) and $P\left(d h g^{f / f} ; P V\right.$-Cre animals (white). Bars show mean \pm SEM from three animals per genotype. $p=0.46$, unpaired $t$ test. Right, Coronal sections with PV+ labeling of SSC of control and Pcdhg ${ }^{f f f}$; PV-Cre animals; ${ }^{*} p<0.05,{ }^{* *} p<0.01,{ }^{* * *} p<0.001$. Scale bars: $50 \mu \mathrm{m}(\boldsymbol{A}), 100 \mu \mathrm{m}(\boldsymbol{C}-\boldsymbol{E})$, and $200 \mu \mathrm{m}(\boldsymbol{F})$.

\section{cIN survival is not dependent on pcdhg expression in other cortical populations}

As a large family of transmembrane molecules with homophilic binding properties, the PCDHGs might mediate cIN survival through cell-cell interactions. We asked whether cIN survival genetically requires $P c d h g$ expression in their afferent/target populations in the cortex: pyramidal neurons, glia, and other cIN subpopulations. Pcdhg ${ }^{f}$ mice were crossed to Emx1-Cre to delete the Pcdhgs from forebrain progenitors that give rise to pyramidal cells and glia, but spare the $P c d h g$ genes in the ganglionic eminence-derived cINs (Fig. 8A). Deletion of Pcdhgs in this model does not affect pyramidal cell survival (Garrett et al., 2012). The densities of mature PV+, SST+ and Reelin+/SST-cIN populations were similar in $P c d h g^{f / f}$; Emx1-Cre mutants to control (Fig. $8 B-D$ ). Therefore, Pcdhgs are not required in pyramidal and glial cell populations to modulate cIN survival.

A compensatory relationship has been proposed between MGE-derived and CGE-derived cINs, in which MGE-cIN reductions are coupled to increased survival of CGE-derived
5HTR3 +/Reelin + interneurons, particularly in the deeper layers (Denaxa et al., 2018). Moreover, VIP + interneurons synaptically target PV+ and SST + interneurons (Pi et al., 2013). To test whether the PCDHGs influence the survival of CGE-cIN nonautonomously, we used Nkx2.1-Cre to delete Pcdhgs from the MGE lineage (Fig. 8F). Similar to ablation with Gad2-Cre, the early postnatal densities of control and Pcdhgff; Nkx2.1-Cre; Ai14-TdTomato mutant populations are initially similar at P2 (1006 cells $/ \mathrm{mm}^{2} \pm 76.5$ cells in Pcdhg ${ }^{W T}$ or Het $; N k x 2.1$-Cre controls vs 1122 cells $/ \mathrm{mm}^{2} \pm 70.9$ cells in Pcdhg f/f $; N k \times 2.1-C r e$ mutants; $N=4$ sections per animal, three to four animals per genotype; $p=0.40$ Mann-Whitney). This finding is consistent with a parallel report showing that the generation and migration of MGE-derived cINs are unaffected in Pcdhg $g^{f / f} ; N k x 2.1-C r e$ mutants (Mancia Leon et al., 2020). By P28, the Cre-ablated TdTomato,$+ \mathrm{SST}+$, and $\mathrm{PV}+$ populations are reduced in Pcdhg f/f; Nkx2.1-Cre mutants compared with control (TdTomato + : $-43.1 \%, p=0.00019$; SST + : $-45.7 \%, p=$ 0.00005 ; PV $+:-25.5 \%, p=0.00019$, unpaired $t$ test from three to four animals per genotype; Fig. $8 G, H)$. However, densities of 
A

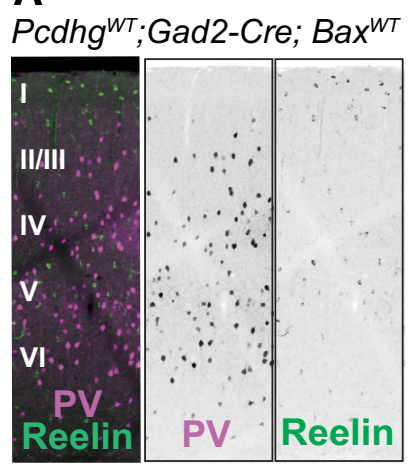

Pcdhgfff; Gad2-Cre; BaxwT
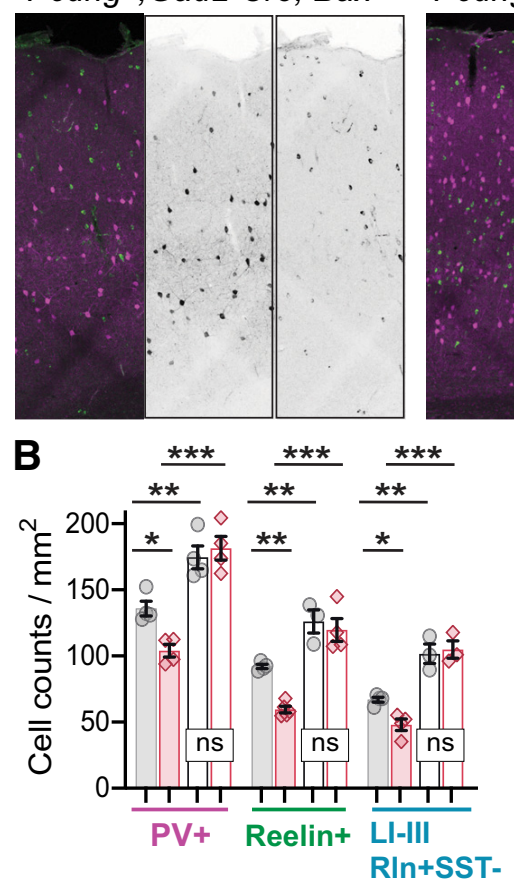
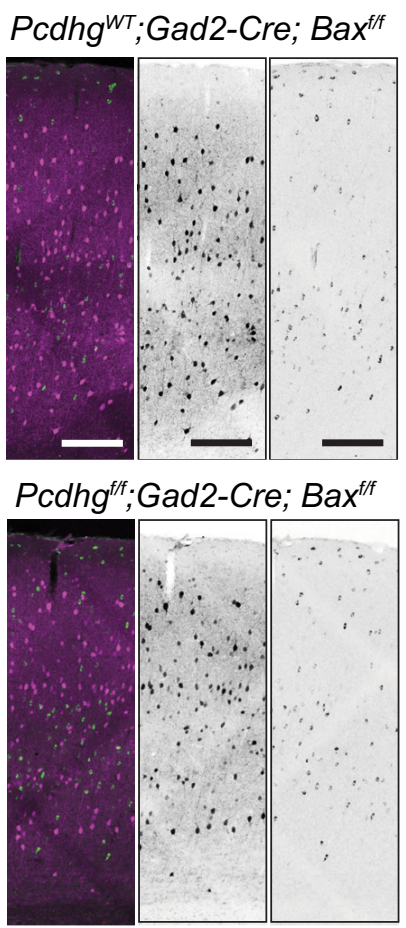

Pcdhg ${ }^{W T} ; B a x^{W T}$ Gad2-Cre

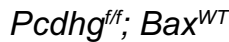
Gad2-Cre

Pcdhg ${ }^{w T} ; B^{f f f}$ Gad2-Cre

Pcdhgff; Bax $^{f f t}$ Gad2-Cre

Figure 6. Genetic blockade of apoptosis by Bax deletion rescues cIN loss in Pcdhg GcKO mutants. A, Immunostaining for PV + (magenta) and Reelin + (green) cINs in S1BF of P28 Pcdhg control Gad2-Cre; Bax ${ }^{+}$(WT or Het), Pcdhg control Gad2-Cre;Bax ${ }^{f / f} ;$ Pcdhg ${ }^{f / f}$;Gad2-Cre; $\mathrm{Bax}^{+}$mutant; and Pcdhg ${ }^{\mathrm{f} / f}$;Gad2-Cre;Bax ${ }^{\text {f/f }}$ double mutant animals. Inverted images of $\mathrm{PV}+$ in center, and Reelin + on right. Reelin labels CGE-derived Reelin +/SST- in LI-III, and MGE-derived cINs that overlap with SST + in LII-LVI. B, Quantifications of total PV+ and Reelin + cINs across layers, and Reelin +/SST- in LI-III. Scatter bar plots show data, mean \pm SEM, from three to four three to four animals per genotype. One-way ANOVA, PV+: $F=24.8 p=0.00002$. Reelin+: $F=30.5 p<0.00001$. Reelin+/SST-: $F=30.9$ $p=0.00002$ Holm-Sidak's pairwise comparisons: $P c d h g^{+} ; G a d 2-C r e ; B a x^{+}$versus $P c d h g^{+}$; Gad2-Cre;Bax ${ }^{f / f}: \mathrm{PV}+p=0.018$; Reelin $+p=0.0059 ; \mathrm{LI}-\mathrm{III} \mathrm{R}+/ \mathrm{SST}-p=0.0019$. Pcdhg ${ }^{\mathrm{f} / \mathrm{f}}$; Gad2-Cre; Bax ${ }^{+}$versus Pcdhg ${ }^{\text {f/f }}$;Gad2-Cre;Bax ${ }^{\mathrm{f} / \mathrm{F}}: \mathrm{PV}+p=0.00004$; Reelin,$+ p=0.00002$; LI-III R+/SST- $p=0.00007$. Pcdhg ${ }^{+} ;$Gad2-Cre;Bax $^{\text {f/f }}$ versus Pcdhg ${ }^{\text {f/f }} ;$ Gad2-Cre;Bax $^{\text {f/ff }}: \mathrm{PV}+$ $p=0.52$; Reelin+ $p=0.47$; LI-III R+/SST- $p=0.67$, ${ }^{*} p<0.05,{ }^{* *} p<0.01$, ${ }^{* * *} p<0.001$. Scale bars: $200 \mu \mathrm{m}$.

CGE-derived Reelin+/SST- and VIP + cINs in the upper and lower layers are unaffected (Fig. 8I). Thus, the sizes of the $\mathrm{Pcdhg}^{+}$-expressing CGE populations are not influenced by nonautonomous effects from Pcdhg-deficient MGE-cINs.

PCDHGs regulate a similar program of interneuron survival in the cerebellar cortex

Finally, we addressed whether the PCDHG pro-survival role extends to GABAergic interneuron populations in other brain

regions. We focused on the cerebellar MLIs which are reduced in Pcdhg GcKO mutants (Fig. 2E). MLIs originate from a Ptf1aexpressing GABAergic lineage in the ventricular zone of the cerebellar primordium (Hoshino et al., 2005). Developmental apoptosis spans from P5 to P15, and occurs during proliferation and settling of Pax2+ MLIs in the molecular layer (Yamanaka et al., 2004; Sergaki et al., 2017). We confirmed Gad2-Cre recombination in MLIs in the postnatal cerebellar cortex with Ai14-TdTomato (Fig. $9 A$ ), and quantified them through during development. Similar to forebrain cIN populations, MLI densities in Pcdhg GcKO animals are initially similar to control but are significantly reduced by P15 $(-22.9 \%, p=0.000014$, unpaired $t$ test; Fig. $9 A, B)$. As the thickness of the molecular layer increases nearly fivefold in this timeframe (Yamanaka et al., 2004), we compared changes in MLI populations by normalizing to the number of Purkinje cells, which are established in a monoplanar arrangement by P6 (Fujita et al., 2012). In Pcdhg controls, MLI populations decline from P11 to P25 (one-way ANOVA, $F_{(9,29)}=57.42 ; P c d h g^{W T}$ or Het $\mathrm{P} 11$ vs P25, $p=0.00075$, Tukey's pairwise comparison). MLIs in Pcdhg GcKO mutants follow a similar pattern of elimination ( $p=0.00006$, post hoc Tukey) but are lost to a greater extent by P15 (Fig. $9 C$ ).

Similar to cINs, declining MLI populations in Pcdhg GcKO mutants coincide with enhanced apoptosis (control, $4.1 \pm 0.3$; Pcdhg GcKO, 12.6 \pm 0.7 CC3/9+ cells $/ \mathrm{mm}^{2}, p<0.00001$, Mann-Whitney; Fig. 9D). To test whether the Pcdhgs are required during the wave of $\mathrm{PCD}$, we used $P V$-Cre which is active in MLIs at a later postnatal stage, as confirmed by the expression of Ai14-TdTomato in MLIs that reach their final position and begin to express PV (Simat et al., 2007; Fig. 9E). MLI densities are unaffected in Pcdhg ${ }^{\text {flf; }}$ PV-Cre mutants (Fig. 9F), further supporting a requirement for the Pcdhgs during the critical period of PCD. Moreover, naturally-occurring MLI death in control and elevated MLI death in Pcdhg GcKO cerebella are Bax dependent (Fig. 9G,H). MLI densities are increased in constitutive Bax KO animals compared with wild type and are indistinguishable in Pcdhg GcKO; Bax KO double mutants compared with $\mathrm{Pcdhg}^{+}$; Bax KO siblings $\left(\mathrm{Pcdhg}^{+}\right.$; Bax ${ }^{+}, 78.8 \pm 4.7$ cells/0.1 $\mathrm{mm}^{2}$ vs Pcdhg ${ }^{+}$; Bax KO, 98.2 $\pm 3.7, p=0.04 ;$ Pcdhg GcKO; $\mathrm{Bax}^{+}, 51.3 \pm 4.3$ vs Pcdhg GcKO; Bax KO, 97.0 $\pm 3.7, p=$ 0.00002; $\mathrm{Pcdhg}^{+}$; Bax KO vs Pcdhg GcKO; Bax KO p =0.99, Tukey pairwise comparisons, four animals per genotype; Fig. $9 G$, $H$ ). Altogether, our findings demonstrate that the PCDHGs promote the survival of two unrelated GABAergic interneuron populations during postnatal PCD. We propose that the PCDHGs act broadly to control survival and final interneuron numbers for multiple populations in the brain.

\section{Discussion}

The pruning of interneuron populations by developmental cell death is critical for establishing balanced excitatory-inhibitory ratios. Recent studies have identified the extent, timing and activity-dependent factors that modulate cIN PCD (Wong and Marín, 2019). A major gap was identification of cell surface molecules that select cINs for survival in ways perhaps analogous to neurotrophin receptors in the PNS. In this study, we identified the PCDHGs as essential regulators of cIN survival that balance the extent of PCD. During the postnatal period when $20-40 \%$ of cINs are naturally eliminated (Southwell et al., 2012; Priya et al., 2018), a further $30-50 \%$ of the population is lost on Pcdhg deletion because of elevated apoptosis. Both natural and Pcdhg-deficient cIN loss are mitigated by conditional deletion of $B a x$, illustrating the pro-survival role for the 
A

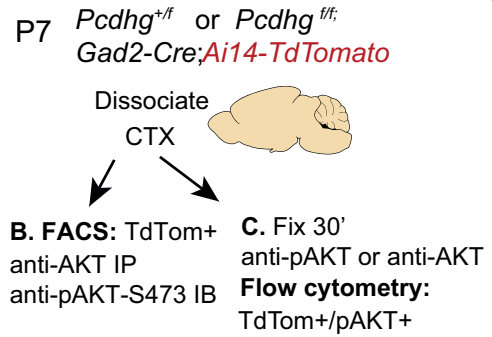

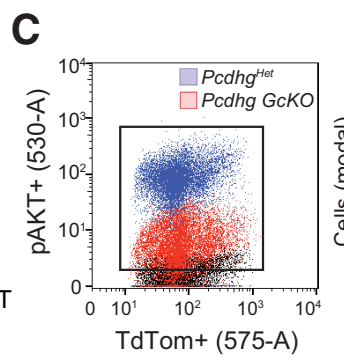
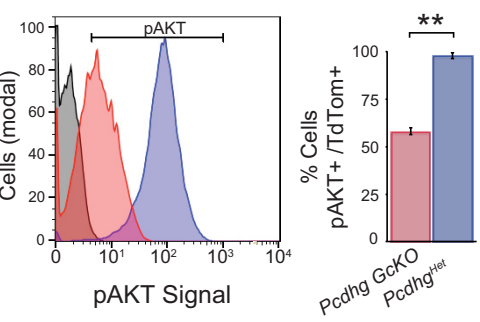
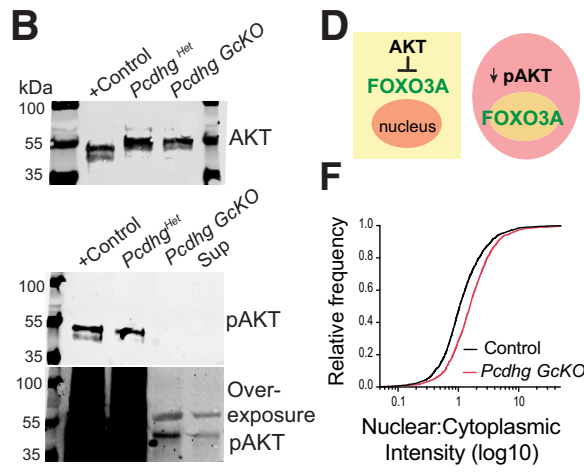

\section{E}
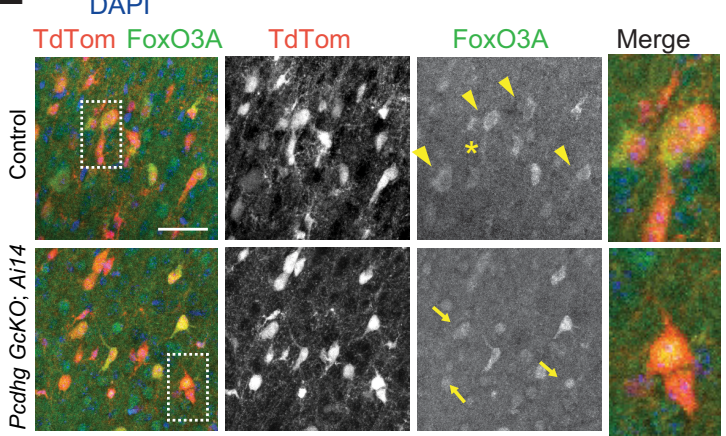

Figure 7. Akt-Fox03A signaling is diminished in Pcdhg GcKO mutants. $\boldsymbol{A}$, Schematic of experimental design for FACS-immunoblot (data in $\boldsymbol{B}$ ) and flow cytometry analysis (data in $\boldsymbol{C}$ ) of pAKT signals in cINs dissociated from P7 cortices. $\boldsymbol{B}$, Immunoblots of AKT (top) and pAKT-Ser473 (middle; bottom, overexposure) following AKT-mediated immunoprecipitation of FACS-sorted Gad2Cre; TdTomato + cortical cells isolated from P7 Pcdhg ${ }^{\text {Het }}$ and Pcdhg GcKO brains. Positive control is a validated sample. Two animals per genotype. Equal numbers of TdTomato + cells from FACS were assayed. Sup, supernatant. C, Cytometric measure of pAKT-immunolabeled Tdtomato + cortical cells isolated from Pcdhg GcKO (red) and heterozygous Pcdhg control (blue). Left, pAKT + (530-A) versus TdTom + (575-A) events from GcK0 and Het samples. Black box $=$ pAKT + event gate; black dots $=$ isotype control. Middle, Distribution of pAKT + events TdTom + population; black line $=$ pAKT + event gate. Mean TdTom + events: $9949.6 \pm 310.4$, Het $=10115 \pm 368.7 ; p=0.75$. Mean pAKT + events: GcKO $=6502 \pm 138$, Het $=9650.3 \pm 321$; $p=0.0008$, from three independent experiments. Right, pAKT + events plotted as percentage of TdTom + cell population. $p=0.0046$, unpaired $t$ test; six brains per genotype. $D$, Schematic of AKT-dependent inhibition of Fox03A translocation from cytoplasmic (top) to nuclear (bottom) compartments. $E$, Immunostaining of P7 cortex shows translocation of Fox03A (green) in Gad2Cre-TdTomato + cINs (red) from a predominantly cytoplasmic localization in control (top, yellow arrowheads) to nuclear enrichment in Pcdhg GcKO mutants (bottom, yellow arrows). Right, High magnification of inset. $\boldsymbol{F}$, Cumulative distribution plot of nuclear to cytoplasmic ratios of Fox03A fluorescence intensities quantified in control (black) and Pcdhg GcKO (red) TdTomato + cINs across Layers II-VI. $N=3$ animals per genotype, $p<0.00001$, KS test; ${ }^{* *} p<0.01,{ }^{* * *} p<0.001$. Scale bar: $25 \mu \mathrm{m}$.

PCDHGs in the PCD pathway. Although Gad2-Cre is active in small numbers of astrocytes, we confirmed the specificity of cIN death to Pcdhg loss-of-function in GABAergic neurons with the Nkx2.1-Cre and Emx1-Cre lines. Our findings are supported by a complementary study, which also demonstrates that, unlike the essential role for the Pcdhg cluster, the Pcdh- $\alpha$ and $P c d h-\beta$ clusters are dispensable for cIN survival (Mancia Leon et al., 2020). By extending the survival role to cerebellar interneurons, our study indicates a broad role for the PCDHGs in controlling the size of GABAergic interneuron populations in the developing brain.

\section{The PCDHGs mediate a pro-survival mechanism for GABAergic interneuron PCD}

The period of cIN PCD is intrinsically-determined, as heterochronically grafted cINs undergo apoptosis according to their intrinsic PCD timing rather than of the host (Southwell et al., 2012). In spinal cord, excess interneuronal death in Pcdhg mutants also follows the exquisite, cell type-specific ordering of PCD (Prasad et al., 2008). Thus, the PCDHGs regulate the extent but not the timing of interneuron PCD. The wave of MGEderived cIN PCD slightly precedes that of the CGE-cINs (Priya et al., 2018), suggesting that cIN susceptibility to cell death is tuned to birth date and cell type. Soon after, the pro-apoptotic machinery is likely downregulated to terminate the wave of PCD (Hollville et al., 2019) and to stabilize final cIN populations for circuit maturation. The intrinsic programs that determine the
PCD timeline and responses to pro-survival or pro-death signals remain unknown.

We propose that the PCDHGs promote cIN survival through modulation of AKT signaling, which is elevated during the peak of PCD (Wong et al., 2018). The PI3K-AKT pathway regulates neuronal survival through multiple anti-apoptotic and pro-survival actions (Brunet et al., 2001). One link between diminished AKT signaling and increased apoptosis in Pcdhg GcKO cINs is the translocation of FOXO3A, which upregulates expression of pro-apoptotic factors such as Puma and Bim (Li et al., 2009; Ambacher et al., 2012). PI3K-AKT promote cortical and hippocampal neuron survival by integrating numerous upstream signals, including trophic factors, spontaneous depolarization and synaptic transmission, and intracellular calcium signaling (Hardingham et al., 2002; Heck et al., 2008; Léveillé et al., 2010; Golbs et al., 2011; Murase et al., 2011; WagnerGolbs and Luhmann, 2012). Neural activity influences cIN survival in a class-specific manner (Denaxa et al., 2018; Priya et al., 2018), while the PCDHGs function in all classes examined. Whether the PCDHGs intersect with activity-dependent mechanisms remains to be determined. One possibility is that PCDHG-dependent and activity-dependent signals converge onto PI3K-AKT or Ca2+-calcineurin-NFAT pathways (Priya et al., 2018).

How do the PCDHGs transduce pro-survival AKT signaling at the membrane? One pro-apoptotic candidate is PTEN, which negatively regulates $\mathrm{PI} 3 \mathrm{~K}$ and is required for CIN PCD (Wong et al., 2018). A pro-survival candidate is PDK1, which 

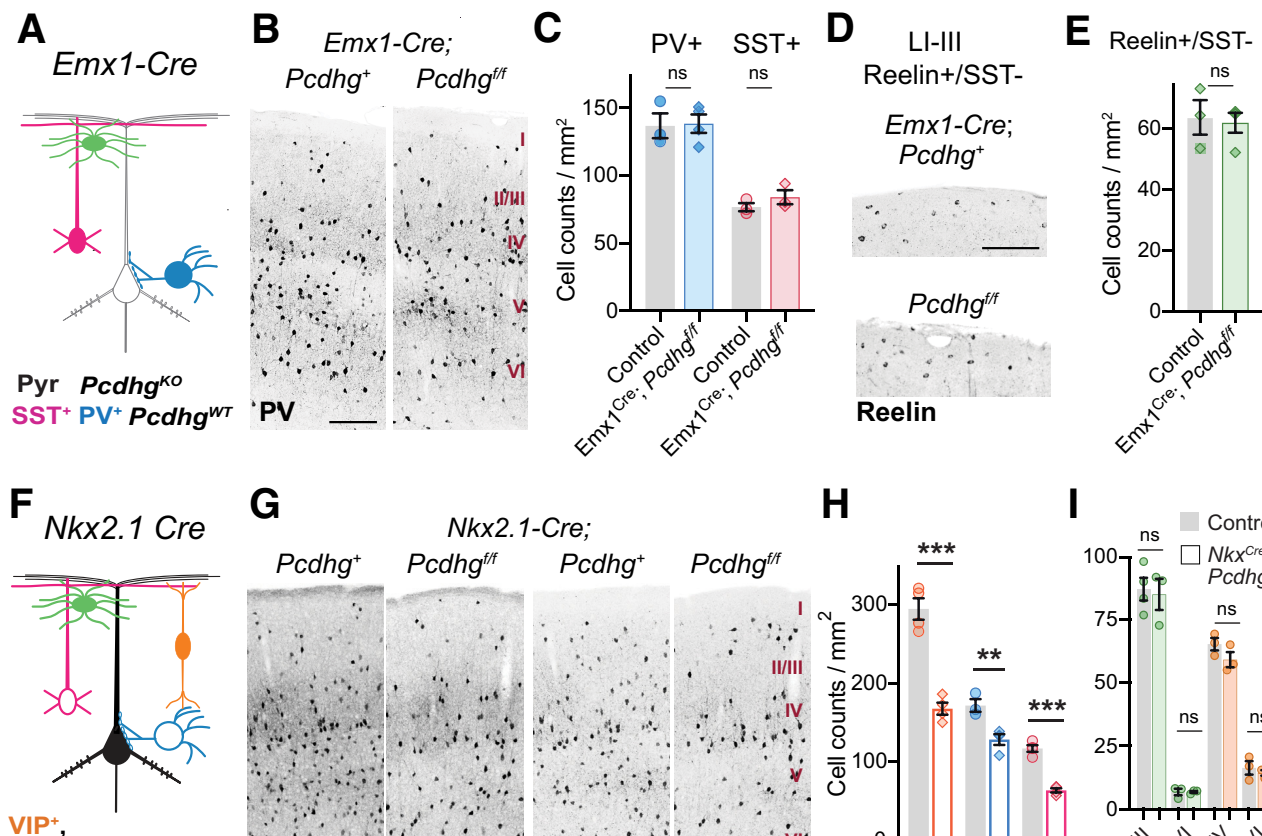

\section{G}

RLN+SST- Pcdhg WT $^{\text {T }}$
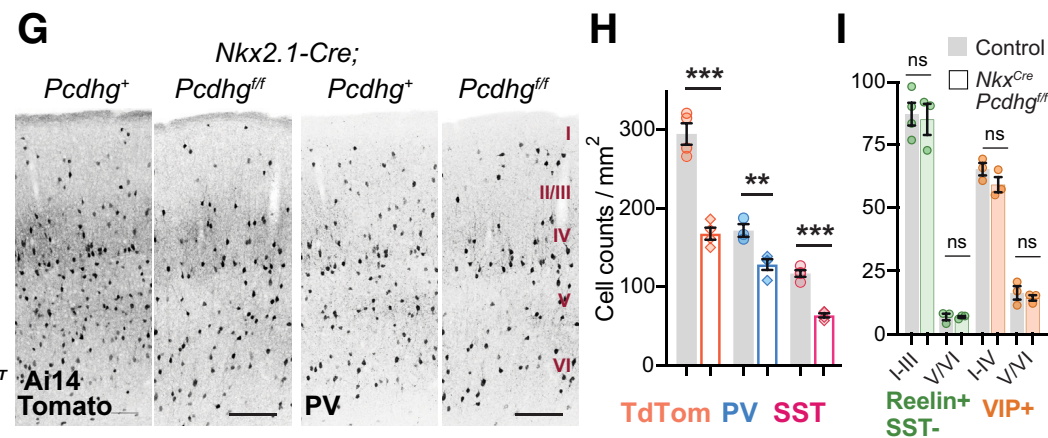

Figure 8. $\quad$ IN survival does not require the $P c d h g s$ in other cortical populations. $A-E$, Test for Pcdhgs in pyramidal population: Emx1-Cre deletes $P c d h g s$ from pyramidal and glial cells, leaving the Pcdhgs intact in cINs. $\boldsymbol{B}$, Inverted fluorescent image of PV + cINs in control and $P\left(d h g^{f / f} ;\right.$ Emx1-Cre mutants at P28. C, Quantifications show that PV $+(p=0.89$, unpaired $t$ test) and SST+ cINs $(p=0.29)$ are not significantly different (ns) in Pcdhg ${ }^{f / f} ;$ Emx1-Cre mutants compared with control. $\boldsymbol{D}$, Inverted image of Layer I CGE-Reelin + cINs in control and Pcdhg ${ }^{f / f} ;$ Emx1-Cre mutants. $\boldsymbol{E}$, Quantifications of Reelin +/SST- cINs in Layers I-III in control and $P\left(d h g^{f f f} ;\right.$ Emx1-Cre mutants (shaded green). Data in $\boldsymbol{C}$, $\boldsymbol{E}$ show mean \pm SEM from three to four animals per genotype, $p=0.78$, unpaired $t$ test. $\boldsymbol{F}-\boldsymbol{I}$, Test for non-autonomous role in (GE-derived Reelin+/SST- (green), using Nkx2.1-Cre to delete Pcdhg fr/f from MGE-derived PV + (blue) and SST+ (pink) cINs. G, Inverted fluorescent images of TdTomato + (left) and PV + (right) cINs of control and Pcdhg ${ }^{\text {t/f }}$;Nkx2.1 1 (re; Ai14-TdTomato animals. H, Quantifications of MGE-derived Tomato + $(p=0.00019), \mathrm{PV}+(p=0.0097)$, and SST $+\left(p=0.00005\right.$, unpaired $t$ test) populations in control (gray bar) and Pcdhg ${ }^{f / f}$; Nkx2.1-Cre mutants (outlined bar). $I$, Quantifications of CGE-derived Reelin+/SST- cINs (green) in Layers I-III ( $p=0.58)$ and Layers V-VI $(p=0.93$, unpaired $t$ test); and VIP + cINs (orange) in Layers I-IV $(p=0.20)$ and Layers V-VI $(p=0.52$, unpaired $t$ test). Data in $\boldsymbol{H}$, I show mean \pm SEM from three to four animals per genotype; ${ }^{* *} p<0.01,{ }^{* * *} p<0.001$. Scale bars: $200 \mu \mathrm{m}$.

positively regulates AKT by phosphorylation. Interneuronspecific ablation of PDK1 also results in enhanced cIN loss (Wei et al., 2020). PYK2 and FAK are negatively regulated by the $\mathrm{CPCDHs}$ in pyramidal neuron migration and arborization (Garrett et al., 2012; Fan et al., 2018), and overexpression of PYK2 induces apoptosis in chick spinal cord (Chen et al., 2009). All PCDHG isoforms share an identical cytoplasmic terminus but bear unique juxtamembrane domains; both domains regulate trafficking (Fernández-Monreal et al., 2009; O’Leary et al., 2011; Shonubi et al., 2015).

\section{Which PCDHG-dependent interactions promote cIN survival?}

The purpose of cIN PCD is presumed to numerically or functionally match inhibitory interneurons to the excitatory pyramidal populations. Given that increasing the activity or numbers of pyramidal neurons expands the surviving cIN population (Wong et al., 2018), one attractive hypothesis is that the cPCDHs mediate homophilic, cell-cell interactions between cINs and pyramidal neurons. In the simplest test of this scenario, we ruled out a requirement for Pcdhgs in pyramidal neurons for cIN survival. This aligns with findings in retina where synaptic targeting are not prerequisites for survival (Lefebvre et al., 2008), although the PCDHGs influence both synaptic contacts and survival in the spinal cord (Weiner et al., 2005). Our results do not account for potential redundancy among the cPCDHs.

A second possibility is that the PCDHGs mediate interactions between cINs to enhance the electrical coupling or GABAergic assemblies that emerge during the first postnatal week (Minlebaev et al., 2011; Pangratz-Fuehrer and Hestrin, 2011; Modol et al., 2020). The abnormal network activities observed in the hippocampus and spinal cord of $c P c d h$ mutants suggest roles in patterning network topology (Hasegawa et al., 2017; Asai et al., 2020). Network activities that influence cIN survival are dependent on GABAergic signaling onto pyramidal neurons leading to cIN death (Duan et al., 2020). Mechanistic investigations are needed to understand how selective interactions mediated by the PCDHGs, and by cell adhesion molecules in other systems (DSCAMS, Dpr/DIPs; Li et al., 2015; Xu et al., 2018), modulate cell death to control interneuron network size and activities during circuit formation.

\section{The PCDHGs mediate a pro-survival program for interneurons throughout the developing CNS}

The unique requirement for the Pcdhgs in cIN survival (Mancia Leon et al., 2020) is consistent with studies in retina and spinal cord (Hasegawa et al., 2016; Ing-Esteves et al., 2018). There, GABAergic and glutamatergic interneuron populations are reduced by roughly half in Pcdhg mutants (Wang et al., 2002; Lefebvre et al., 2008; Prasad et al., 2008). Of the 22 Pcdhgs, deletion of the three Pcdhgc3, Pcdhgc4, and Pcdhgc5 genes recapitulates the cell death phenotypes in retina, spinal cord (Chen et al., 2012) and among cINs (Mancia Leon et al., 2020). As revealed by CRISPR-induced deletions across the Pcdhg cluster, Pcdhgc4 alone is essential for spinal and retinal neuron survival (Garrett et al., 2019). However, the remaining $c P c d h s$ contribute to 


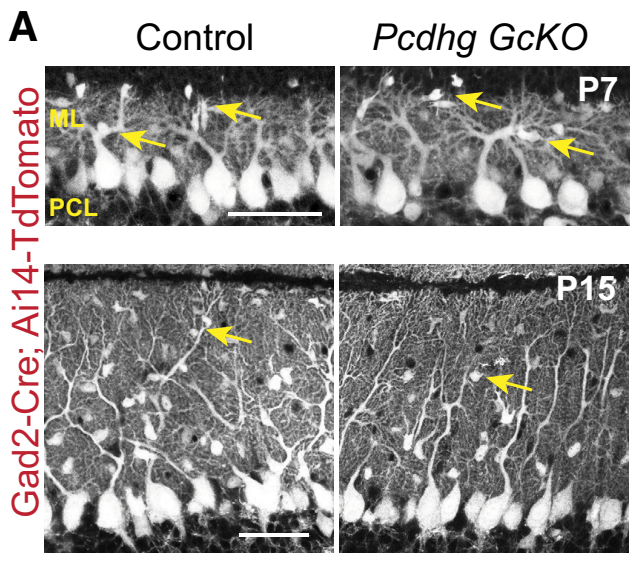

D cc3/9 TdTomato
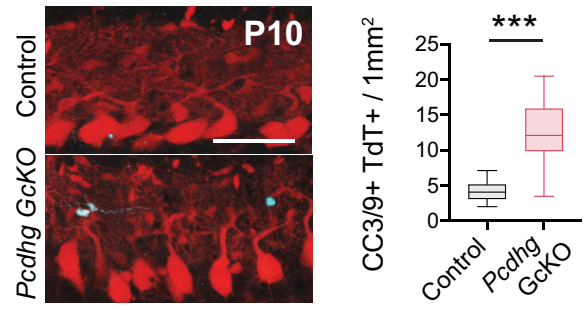

B

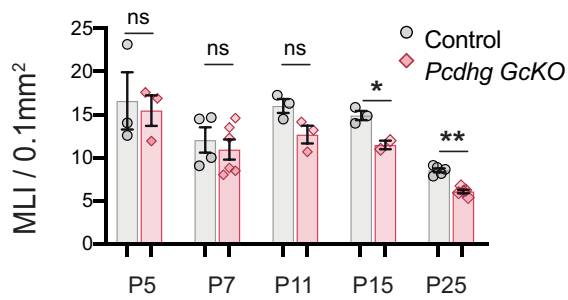

C

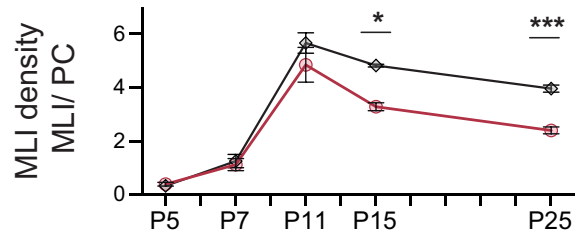

$\mathbf{E}$

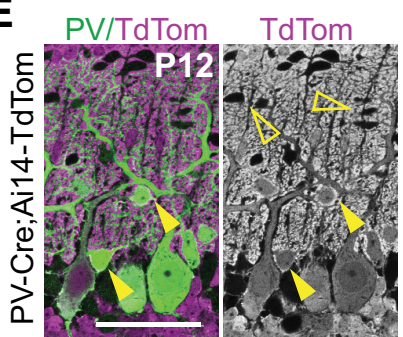

$\mathbf{F}$

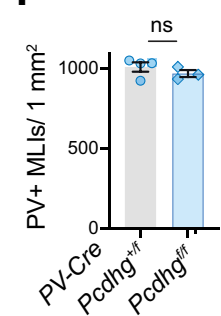

\section{G} Pcdhg $^{+}$Gad2-Cre; Pcdhg ${ }^{f f f}$ Gad2-Cre; Pcdhg ${ }^{+}$Gad2-Cre; Pcdhg ${ }^{f f f}$ Gad2-Cre;
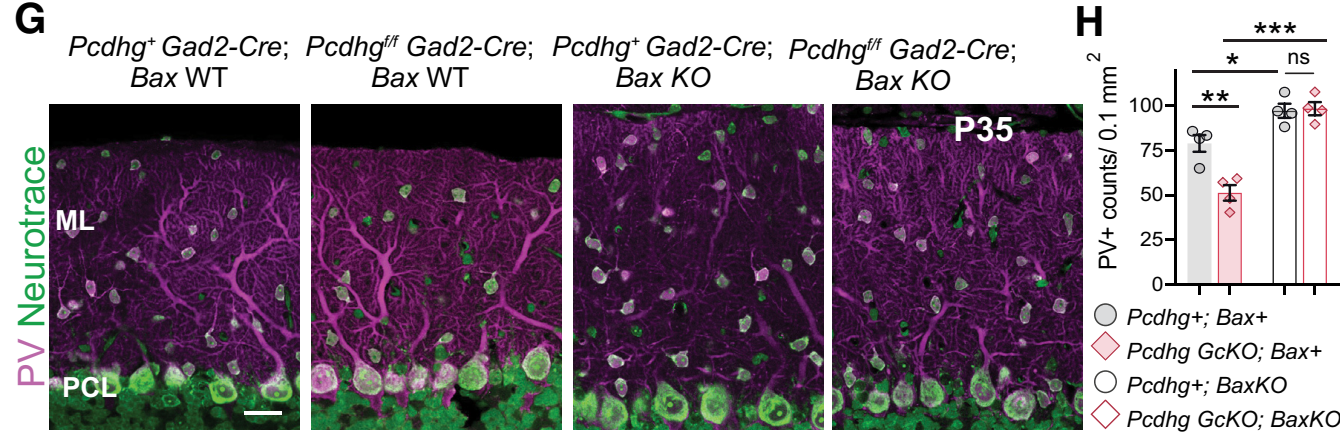

Figure 9. Pcdhgs promote the survival of GABAergic cerebellar interneurons during PCD. A, MLls labeled by Gad2-(re; Ai14-TdTomato in control and Pcdhg GcKO cerebellar cortex at P7 (top) and P15 (bottom). Numbers of MLIs (yellow arrows) increase postnatally in the molecular layer (ML). TdTomato+ soma of Purkinje cells are located in the Purkinje cell layer (PCL). $\boldsymbol{B}$, Quantification of MLIs in the ML in control (gray) and Pcdhg GCKO mutants (red), marked by TdTomato (P5, P7, P15), or PAX2+ and PV+ (P11, P25). Bars show data, mean \pm SEM from three to five animals per genotypes (two for P15 GCKO). P5 $p=0.98 ; \mathrm{P} 7 p=0.48 ; \mathrm{P} 11 p=0.1 ; \mathrm{P} 15 p=0.021 ; \mathrm{P} 15, p=0.008$, Mann-Whitney $U$ test. C, MLI density over time, represented by MLI counts normalized by the numbers of PCS. P5 $p=0.34 ; \mathrm{P} 7 p=0.67$; P11 $p=0.35$; P15 $p=0.04 ; \mathrm{P} 25, p<0.00001$, unpaired $t$ test with Welch's correction. $\boldsymbol{D}$, Left, Immunostaining of TdTomato + MLls (red) with anti-CC3 and anti-CC9 (cyan) in control and Pcdhg GcKO cerebellar cortex at P10. Right, Quantifications of CC3/9+/TdTomato + MLls. Box plots show data from eight sections per animal, four animals per Pcdhg control or GCKO group; $p<0.00001$, Mann-Whitney U test. E, PV-Cre;Ai14-TdTomato recombination pattern at P12 shows TdTomato + MLIs (magenta, yellow filled arrowhead) co-labeled with anti-PV (green) in the lower ML, and TdTomato-negative MLIs in the upper ML (yellow outline arrowhead). $\boldsymbol{F}$, Quantifications of cerebellar PV+/NT+ interneurons in Pcdhg PV-Cre animals. Bars show data, mean \pm SEM from three animals per genotypes $p=0.31$, unpaired $t$ test with Welch's correction. G, MLLs marked by PV (magenta) and NeuroTrace (green) from P35 Pcdhg;Gad2-Cre animals crossed to constitutive BaxKO allele. $\boldsymbol{H}$, Quantifications of PV+/NT+ MLIs in the ML. Bars show mean \pm SEM and counts from four animals per genotype. ANOVA, $F=27.3 p=0.000012$. Post hoc Tukey's pairwise comparison tests: $P$ cdhg ${ }^{+} ;$Gad2-Cre;Bax $^{+}$versus Pcdhg ${ }^{+}$;Gad2-Cre;BaxKO: $p=0.04$. Pcdhg ${ }^{\text {fff }}$;Gad2Cre;Bax ${ }^{+}$versus Pcdhg ${ }^{\text {ffff }}$ Gad2-Cre;BaxKO: $p=0.000022$. Pcdhg ${ }^{+}$;Gad2-Cre;BaxKO versus Pcdhg ${ }^{\text {ffff }}$ Gad2-Cre;BaxKO: $p=0.99,{ }^{*} p<0.05,{ }^{* *} p<0.01,{ }^{* * *} p<0.001$. Scale bars: $50 \mu \mathrm{m}(\boldsymbol{A}, \mathbf{D}$, G) and $25 \mu \mathrm{m}(\boldsymbol{F})$.

survival, albeit with partial redundancy, because removal of $P c d h a$ and/or Pcdhb in addition to Pcdhg augments interneuron loss to $80-95 \%$ (Hasegawa et al., 2016; Ing-Esteves et al., 2018). Whether the surviving cINs in Pcdhg mutants are also dependent on PCDHAs and PCDHBs remains to be tested. Alternatively, cIN subpopulations might be regulated by other survival pathways, or be refractory to PCD altogether. Similar cooperative survival roles are also observed between neurotrophins/Trks in the PNS. For instance, survival of sympathetic ganglia is exclusively dependent on NGF-TrkA and deletion of either gene causes a complete neuronal loss. By contrast, loss of sensory ganglionic subpopulations is additive with deletion of multiple ligands or receptors (Huang and Reichardt, 2001). Interestingly, GDNF receptors RET and GFR1a promote cerebellar interneuron survival during PCD (Sergaki et al., 2017), raising the possibility of co-signaling between CPCDHs and RET (Schalm et al., 2010). Delineating interneuron-specific CPCDH roles in survival requires mapping isoform-specific expression and functions, including PCDHGC4-specific actions.

Although sensory and principal neurons are largely unaffected in Pcdhg mutants (Wang et al., 2002; Garrett et al., 2012; Lefebvre et al., 2012), PCDHG-dependent survival is not exclusive to interneurons. The exception to date are the retinal ganglion cells (Lefebvre et al., 2008). The volume reductions of 
GABAergic neuron-rich regions in Pcdhg GcKO brains suggest a role in the survival of GABAergic projection populations, such as those in the GP which undergo PCD (Waters et al., 1994). In the hypothalamus, increased developmental apoptosis in Pcdhg mutants occurs in regions containing glutamatergic and GABAergic projection neurons (Su et al., 2010).

In conclusion, the PCDHGs provide a molecular basis for controlling the size of GABAergic interneuron populations. The neurologic deficits and spontaneous seizures displayed by $P c d h g$ GcKO mutants underscore the importance of PCDHGs for development of excitatory-inhibitory balance and circuit function. The cortical epileptiform activities might be explained by cIN loss or reduced GABA neurotransmission, as in other models of developmental interneuron deficits (Cobos et al., 2005; Lin et al., 2018). Hindlimb clasping likely results from GABAergic deficits in multiple circuits controlling sensorimotor function (Lalonde and Strazielle, 2011). Additional experiments are needed to determine whether dysregulation of excitation-inhibition in different interneuron populations underlie circuit phenotypes in $P c d h g$ GcKO mice. Interneuron deficiency (Kataoka et al., 2010; Lewis et al., 2012), and variants and dysregulated expression of the human cPCDH gene clusters are associated with neurodevelopmental and psychiatric disorders, including Autism spectrum disorder, Tourette's, Down's, and schizophrenia (Iossifor et al., 2012; Anitha et al., 2013; El Hajj et al., 2016; Shao et al., 2019; Vadodaria et al., 2019). These findings have relevance for understanding the developmental basis of inhibitory deficits in human disorders.

\section{References}

Allemang-Grand R, Ellegood J, Spencer Noakes L, Ruston J, Justice M, Nieman BJ, Lerch JP (2017) Neuroanatomy in mouse models of Rett syndrome is related to the severity of Mecp2 mutation and behavioral phenotypes. Mol Autism 8:32.

Ambacher KK, Pitzul KB, Karajgikar M, Hamilton A, Ferguson SS, Cregan SP (2012) The JNK- and AKT/GSK3 $\beta$ - signaling pathways converge to regulate Puma induction and neuronal apoptosis induced by trophic factor deprivation. PLoS One 7:e46885.

Anitha A, Thanseem I, Nakamura K, Yamada K, Iwayama Y, Toyota T, Iwata Y, Suzuki K, Sugiyama T, Tsujii M, Yoshikawa T, Mori N (2013) Protocadherin $\alpha$ (PCDHA) as a novel susceptibility gene for autism. J Psychiatry Neurosci 38:192-198.

Asai H, Ohkawa N, Saitoh Y, Ghandour K, Murayama E, Nishizono H, Matsuo M, Hirayama T, Kaneko R, Muramatsu SI, Yagi T, Inokuchi K (2020) $\operatorname{Pcdh} \beta$ deficiency affects hippocampal CA1 ensemble activity and contextual fear discrimination. Mol Brain 13:7.

Avants B, Duda JT, Kim J, Zhang H, Pluta J, Gee JC, Whyte J (2008) Multivariate analysis of structural and diffusion imaging in traumatic brain injury. Acad Radiol 15:1360-1375.

Avants BB, Tustison NJ, Song G, Cook PA, Klein A, Gee JC (2011) A reproducible evaluation of ANTs similarity metric performance in brain image registration. Neuroimage 54:2033-2044.

Blanquie O, Yang JW, Kilb W, Sharopov S, Sinning A, Luhmann HJ (2017) Electrical activity controls area-specific expression of neuronal apoptosis in the mouse developing cerebral cortex. Elife 6:e27696.

Brunet A, Datta SR, Greenberg ME (2001) Transcription-dependent and -independent control of neuronal survival by the PI3K-Akt signaling pathway. Curr Opin Neurobiol 11:297-305.

Cahill LS, Laliberté CL, Ellegood J, Spring S, Gleave JA, Eede MC, Lerch JP, Henkelman RM (2012) Preparation of fixed mouse brains for MRI. Neuroimage 60:933-939.

Chen J, Lu Y, Meng S, Han MH, Lin C, Wang X (2009) Alpha- and gammaprotocadherins negatively regulate PYK2. J Biol Chem 284:2880-2890.

Chen WV, Alvarez FJ, Lefebvre JL, Friedman B, Nwakeze C, Geiman E, Smith C, Thu CA, Tapia JC, Tasic B, Sanes JR, Maniatis T (2012) Functional significance of isoform diversification in the protocadherin gamma gene cluster. Neuron 75:402-409.

Chen WV, Nwakeze CL, Denny CA, O'Keeffe S, Rieger MA, Mountoufaris G, Kirner A, Dougherty JD, Hen R, Wu Q, Maniatis T (2017a) Pcdh $\alpha$ c2 is required for axonal tiling and assembly of serotonergic circuitries in mice. Science 356:406-411.

Chen YJ, Friedman BA, Ha C, Durinck S, Liu J, Rubenstein JL, Seshagiri S, Modrusan Z (2017b) Single-cell RNA sequencing identifies distinct mouse medial ganglionic eminence cell types. Sci Rep 7:45656.

Choi HM, Chang JY, Trinh le A, Padilla JE, Fraser SE, Pierce NA (2010) Programmable in situ amplification for multiplexed imaging of mRNA expression. Nat Biotechnol 28:1208-1212.

Cobos I, Calcagnotto ME, Vilaythong AJ, Thwin MT, Noebels JL, Baraban SC, Rubenstein JL (2005) Mice lacking Dlx1 show subtype-specific loss of interneurons, reduced inhibition and epilepsy. Nat Neurosci 8:10591068.

de Guzman AE, Wong MD, Gleave JA, Nieman BJ (2016) Variations in postperfusion immersion fixation and storage alter MRI measurements of mouse brain morphometry. Neuroimage 142:687-695.

Denaxa M, Neves G, Rabinowitz A, Kemlo S, Liodis P, Burrone J, Pachnis V (2018) Modulation of apoptosis controls inhibitory interneuron number in the cortex. Cell Rep 22:1710-1721.

Dorr AE, Lerch JP, Spring S, Kabani N, Henkelman RM (2008) High resolution three-dimensional brain atlas using an average magnetic resonance image of 40 adult C57Bl/6J mice. Neuroimage 42:60-69.

Duan ZRS, Che A, Chu P, Modol L, Bollmann Y, Babij R, Fetcho RN, Otsuka T, Fuccillo MV, Liston C, Pisapia DJ, Cossart R, De Marco GN (2020) GABAergic restriction of network dynamics regulates interneuron survival in the developing cortex. Neuron 105:75-92.e5.

Ehrlich I, Humeau Y, Grenier F, Ciocchi S, Herry C, Lüthi A (2009) Amygdala inhibitory circuits and the control of fear memory. Neuron 62:757-771.

El Hajj N, Dittrich M, Böck J, Kraus TF, Nanda I, Müller T, Seidmann L, Tralau T, Galetzka D, Schneider E, Haaf T (2016) Epigenetic dysregulation in the developing Down syndrome cortex. Epigenetics 11:563-578.

Fan L, Lu Y, Shen X, Shao H, Suo L, Wu Q (2018) Alpha protocadherins and Pyk2 kinase regulate cortical neuron migration and cytoskeletal dynamics via Rac1 GTPase and WAVE complex in mice. Elife 7:e35242.

Fernández-Monreal M, Kang S, Phillips GR (2009) Gamma-protocadherin homophilic interaction and intracellular trafficking is controlled by the cytoplasmic domain in neurons. Mol Cell Neurosci 40:344-353.

Fujita H, Morita N, Furuichi T, Sugihara I (2012) Clustered fine compartmentalization of the mouse embryonic cerebellar cortex and its rearrangement into the postnatal striped configuration. J Neurosci 32:1568815703.

Garrett AM, Schreiner D, Lobas MA, Weiner JA (2012) $\gamma$-Protocadherins control cortical dendrite arborization by regulating the activity of a FAK/ PKC/MARCKS signaling pathway. Neuron 74:269-276.

Garrett AM, Bosch PJ, Steffen DM, Fuller LC, Marcucci CG, Koch AA, Bais P, Weiner JA, Burgess RW (2019) CRISPR/Cas9 interrogation of the mouse Pcdhg gene cluster reveals a crucial isoform-specific role for Pcdhgc4. PLoS Genet 15:e1008554.

Genovese CR, Lazar NA, Nichols T (2002) Thresholding of statistical maps in functional neuroimaging using the false discovery rate. Neuroimage $15: 870-878$

Gilley J, Coffer PJ, Ham J (2003) FOXO transcription factors directly activate bim gene expression and promote apoptosis in sympathetic neurons. J Cell Biol 162:613-622.

Golbs A, Nimmervoll B, Sun JJ, Sava IE, Luhmann HJ (2011) Control of programmed cell death by distinct electrical activity patterns. Cereb Cortex 21:1192-1202.

Gorski JA, Talley T, Qiu M, Puelles L, Rubenstein JL, Jones KR (2002) Cortical excitatory neurons and glia, but not GABAergic neurons, are produced in the Emx1-expressing lineage. J Neurosci 22:6309-6314.

Hardingham GE, Fukunaga Y, Bading H (2002) Extrasynaptic NMDARs oppose synaptic NMDARs by triggering CREB shut-off and cell death pathways. Nat Neurosci 5:405-414.

Hasegawa S, Kumagai M, Hagihara M, Nishimaru H, Hirano K, Kaneko R, Okayama A, Hirayama T, Sanbo M, Hirabayashi M, Watanabe M, Hirabayashi T, Yagi T (2016) Distinct and cooperative functions for the Protocadherin- $\alpha,-\beta$ and $-\gamma$ clusters in neuronal survival and axon targeting. Front Mol Neurosci 9:155.

Hasegawa S, Kobayashi H, Kumagai M, Nishimaru H, Tarusawa E, Kanda H, Sanbo M, Yoshimura Y, Hirabayashi M, Hirabayashi T, Yagi T (2017) Clustered protocadherins are required for building functional neural circuits. Front Mol Neurosci 10:114. 
Heck N, Golbs A, Riedemann T, Sun JJ, Lessmann V, Luhmann HJ (2008) Activity-dependent regulation of neuronal apoptosis in neonatal mouse cerebral cortex. Cereb Cortex 18:1335-1349.

Hernández VM, Hegeman DJ, Cui Q, Kelver DA, Fiske MP, Glajch KE, Pitt JE, Huang TY, Justice NJ, Chan CS (2015) Parvalbumin + neurons and Npas1+ neurons are distinct neuron classes in the mouse external globus pallidus. J Neurosci 35:11830-11847.

Hippenmeyer S, Vrieseling E, Sigrist M, Portmann T, Laengle C, Ladle DR, Arber S (2005) A developmental switch in the response of DRG neurons to ETS transcription factor signaling. PLoS Biol 3:e159.

Hollville E, Romero SE, Deshmukh M (2019) Apoptotic cell death regulation in neurons. FEBS J 286:3276-3298.

Hoshino M, Nakamura S, Mori K, Kawauchi T, Terao M, Nishimura YV, Fukuda A, Fuse T, Matsuo N, Sone M, Watanabe M, Bito H, Terashima T, Wright CV, Kawaguchi Y, Nakao K, Nabeshima Y (2005) Ptfla, a bHLH transcriptional gene, defines GABAergic neuronal fates in cerebellum. Neuron 47:201-213.

Huang EJ, Reichardt LF (2001) Neurotrophins: roles in neuronal development and function. Annu Rev Neurosci 24:677-736.

Ing-Esteves S, Kostadinov D, Marocha J, Sing AD, Joseph KS, Laboulaye MA, Sanes JR, Lefebvre JL (2018) Combinatorial effects of alpha- and gamma-protocadherins on neuronal survival and dendritic self-avoidance. J Neurosci 38:2713-2729.

Iossifov I, Ronemus M, Levy D, Wang Z, Hakker I, Rosenbaum J, Yamrom B, Lee YH, Narzisi G, Leotta A, Kendall J, Grabowska E, Ma B, Marks S, Rodgers L, Stepansky A, Troge J, Andrews P, Bekritsky M, Pradhan K, et al. (2012) De novo gene disruptions in children on the autistic spectrum. Neuron 74:285-299.

Joshi K, Shen L, Cao F, Dong S, Jia Z, Cortez MA, Snead OC 3rd (2018) Kcnj6(GIRK2) trisomy is not sufficient for conferring the susceptibility to infantile spasms seen in the Ts65Dn mouse model of down syndrome. Epilepsy Res 145:82-88.

Kataoka Y, Kalanithi PS, Grantz H, Schwartz ML, Saper C, Leckman JF, Vaccarino FM (2010) Decreased number of parvalbumin and cholinergic interneurons in the striatum of individuals with Tourette syndrome. J Comp Neurol 518:277-291.

Katori S, Hamada S, Noguchi Y, Fukuda E, Yamamoto T, Yamamoto H, Hasegawa S, Yagi T (2009) Protocadherin-alpha family is required for serotonergic projections to appropriately innervate target brain areas. J Neurosci 29:9137-9147.

Kim HY, Yang YR, Hwang H, Lee HE, Jang HJ, Kim J, Yang E, Kim H, Rhim H, Suh PG, Kim JI (2019) Deletion of PLC $\gamma 1$ in GABAergic neurons increases seizure susceptibility in aged mice. Sci Rep 9:17761.

Knudson CM, Tung KS, Tourtellotte WG, Brown GA, Korsmeyer SJ (1995) Bax-deficient mice with lymphoid hyperplasia and male germ cell death. Science 270:96-99.

Kudo T, Uchigashima M, Miyazaki T, Konno K, Yamasaki M, Yanagawa Y, Minami M, Watanabe M (2012) Three types of neurochemical projection from the bed nucleus of the stria terminalis to the ventral tegmental area in adult mice. J Neurosci 32:18035-18046.

Lalonde R, Strazielle C (2011) Brain regions and genes affecting limb-clasping responses. Brain Res Rev 67:252-259.

Lazarus MS, Krishnan K, Huang ZJ (2015) GAD67 deficiency in parvalbumin interneurons produces deficits in inhibitory transmission and network disinhibition in mouse prefrontal cortex. Cereb Cortex 25:1290-1296.

Lefebvre JL (2017) Neuronal territory formation by the atypical cadherins and clustered protocadherins. Semin Cell Dev Biol 69:111-121.

Lefebvre JL, Zhang Y, Meister M, Wang X, Sanes JR (2008) Gamma-protocadherins regulate neuronal survival but are dispensable for circuit formation in retina. Development 135:4141-4151.

Lefebvre JL, Kostadinov D, Chen WV, Maniatis T, Sanes JR (2012) Protocadherins mediate dendritic self-avoidance in the mammalian nervous system. Nature 488:517-521.

Lein ES, Hawrylycz MJ, Ao N, Ayres M, Bensinger A, Bernard A, Boe AF, Boguski MS, Brockway KS, Byrnes EJ, Chen L, Chen L, Chen TM, Chin MC, Chong J, Crook BE, Czaplinska A, Dang CN, Datta S, Dee NR, et al. (2007) Genome-wide atlas of gene expression in the adult mouse brain. Nature 445:168-176.

Lerch JP, Carroll JB, Spring S, Bertram LN, Schwab C, Hayden MR, Henkelman RM (2008) Automated deformation analysis in the YAC128 Huntington disease mouse model. Neuroimage 39:32-39.
Lerch JP, Sled JG, Henkelman RM (2011) MRI phenotyping of genetically altered mice. Methods Mol Biol 711:349-361.

Léveillé F, Papadia S, Fricker M, Bell KF, Soriano FX, Martel MA, Puddifoot C, Habel M, Wyllie DJ, Ikonomidou C, Tolkovsky AM, Hardingham GE (2010) Suppression of the intrinsic apoptosis pathway by synaptic activity. J Neurosci 30:2623-2635.

Lewis DA, Curley AA, Glausier JR, Volk DW (2012) Cortical parvalbumin interneurons and cognitive dysfunction in schizophrenia. Trends Neurosci 35:57-67.

Li D, Qu Y, Mao M, Zhang X, Li J, Ferriero D, Mu D (2009) Involvement of the PTEN-AKT-FOXO3a pathway in neuronal apoptosis in developing rat brain after hypoxia-ischemia. J Cereb Blood Flow Metab 29:19031913.

Li S, Sukeena JM, Simmons AB, Hansen EJ, Nuhn RE, Samuels IS, Fuerst PG (2015) DSCAM promotes refinement in the mouse retina through cell death and restriction of exploring dendrites. J Neurosci 35:5640-5654.

Lin TW, Tan Z, Barik A, Yin DM, Brudvik E, Wang H, Xiong WC, Mei L (2018) Regulation of synapse development by Vgat deletion from ErbB4positive interneurons. J Neurosci 38:2533-2550.

Madisen L, Zwingman TA, Sunkin SM, Oh SW, Zariwala HA, Gu H, Ng LL, Palmiter RD, Hawrylycz MJ, Jones AR, Lein ES, Zeng H (2010) A robust and high-throughput Cre reporting and characterization system for the whole mouse brain. Nat Neurosci 13:133-140.

Mancia Leon WR, Spatazza J, Rakela B, Chatterjee A, Pande V, Maniatis T, Hasenstaub AR, Stryker MP, Alvarez-Buylla A (2020) Clustered gammaprotocadherins regulate cortical interneuron programmed cell death. Elife 9:e55374.

Mayhew TM, Gundersen HJ (1996) If you assume, you can make an ass out of $u$ and me': a decade of the disector for stereological counting of particles in 3D space. J Anat 188:1-15.

Minlebaev M, Colonnese M, Tsintsadze T, Sirota A, Khazipov R (2011) Early $\gamma$ oscillations synchronize developing thalamus and cortex. Science 334:226-229.

Modol L, Bollmann Y, Tressard T, Baude A, Che A, Duan ZRS, Babij R, De Marco García NV, Cossart R (2020) Assemblies of perisomatic GABAergic neurons in the developing barrel cortex. Neuron 105:93-105. e4.

Moffitt JR, Zhuang X (2016) RNA imaging with multiplexed error-robust fluorescence in situ hybridization (MERFISH). Methods Enzymol 572:1-49.

Moffitt JR, Hao J, Wang G, Chen KH, Babcock HP, Zhuang X (2016) Highthroughput single-cell gene-expression profiling with multiplexed errorrobust fluorescence in situ hybridization. Proc Natl Acad Sci USA 113:11046-11051.

Molumby MJ, Keeler AB, Weiner JA (2016) Homophilic protocadherin cellcell interactions promote dendrite complexity. Cell Rep 15:1037-1050.

Mountoufaris G, Chen WV, Hirabayashi Y, O'Keeffe S, Chevee M, Nwakeze CL, Polleux F, Maniatis T (2017) Multicluster Pcdh diversity is required for mouse olfactory neural circuit assembly. Science 356:411-414.

Mountoufaris G, Canzio D, Nwakeze CL, Chen WV, Maniatis T (2018) Writing, reading, and translating the clustered protocadherin cell surface recognition code for neural circuit assembly. Annu Rev Cell Dev Biol 34:471-493.

Murase S, Owens DF, McKay RD (2011) In the newborn hippocampus, neurotrophin-dependent survival requires spontaneous activity and integrin signaling. J Neurosci 31:7791-7800.

O’Leary R, Reilly JE, Hanson HH, Kang S, Lou N, Phillips GR (2011) A variable cytoplasmic domain segment is necessary for $\gamma$-protocadherin trafficking and tubulation in the endosome/lysosome pathway. Mol Biol Cell 22:4362-4372

Pangratz-Fuehrer S, Hestrin S (2011) Synaptogenesis of electrical and GABAergic synapses of fast-spiking inhibitory neurons in the neocortex. J Neurosci 31:10767-10775.

Pfisterer U, Khodosevich K (2017) Neuronal survival in the brain: neuron type-specific mechanisms. Cell Death Dis 8:e2643.

Pi HJ, Hangya B, Kvitsiani D, Sanders JI, Huang ZJ, Kepecs A (2013) Cortical interneurons that specialize in disinhibitory control. Nature 503:521-524.

Prasad T, Wang X, Gray PA, Weiner JA (2008) A differential developmental pattern of spinal interneuron apoptosis during synaptogenesis: insights from genetic analyses of the protocadherin- $\gamma$ gene cluster. Development 135:4153-4164.

Priya R, Paredes MF, Karayannis T, Yusuf N, Liu X, Jaglin X, Graef I, Alvarez-Buylla A, Fishell G (2018) Activity regulates cell death within 
cortical interneurons through a calcineurin-dependent mechanism. Cell Rep 22:1695-1709.

Racine RJ (1972) Modification of seizure activity by electrical stimulation. II. Motor seizure. Electroencephalogr Clin Neurophysiol 32:281-294.

Rudy B, Fishell G, Lee S, Hjerling-Leffler J (2011) Three groups of interneurons account for nearly $100 \%$ of neocortical GABAergic neurons. Dev Neurobiol 71:45-61.

Sahara S, Yanagawa Y, O'Leary DD, Stevens CF (2012) The fraction of cortical GABAergic neurons is constant from near the start of cortical neurogenesis to adulthood. J Neurosci 32:4755-4761.

Saunders A, Huang KW, Sabatini BL (2016) Globus pallidus externus neurons expressing parvalbumin interconnect the subthalamic nucleus and striatal interneurons. PLoS One 11:e0149798.

Schalm SS, Ballif BA, Buchanan SM, Phillips GR, Maniatis T (2010) Phosphorylation of protocadherin proteins by the receptor tyrosine kinase Ret. Proc Natl Acad Sci USA 107:13894-13899.

Schindelin J, Arganda-Carreras I, Frise E, Kaynig V, Longair M, Pietzsch T, Preibisch S, Rueden C, Saalfeld S, Schmid B, Tinevez JY, White DJ, Hartenstein V, Eliceiri K, Tomancak P, Cardona A (2012) Fiji: an opensource platform for biological-image analysis. Nat Methods 9:676-682.

Sergaki MC, López-Ramos JC, Stagkourakis S, Gruart A, Broberger C, Delgado-García JM, Ibáñez CF (2017) Compromised survival of cerebellar molecular layer interneurons lacking GDNF receptors GFR $\alpha 1$ or RET impairs normal cerebellar motor learning. Cell Rep 19:1977-1986.

Shao Z, Noh H, Bin Kim W, Ni P, Nguyen C, Cote SE, Noyes E, Zhao J, Parsons T, Park JM, Zheng K, Park JJ, Coyle JT, Weinberger DR, Straub RE, Berman KF, Apud J, Ongur D, Cohen BM, McPhie DL, et al. (2019) Dysregulated protocadherin-pathway activity as an intrinsic defect in induced pluripotent stem cell-derived cortical interneurons from subjects with schizophrenia. Nat Neurosci 22:229-242.

Shonubi A, Roman C, Phillips GR (2015) The clustered protocadherin endolysosomal trafficking motif mediates cytoplasmic association. BMC Cell Biol 16:28.

Simat M, Ambrosetti L, Lardi-Studler B, Fritschy JM (2007) GABAergic synaptogenesis marks the onset of differentiation of basket and stellate cells in mouse cerebellum. Eur J Neurosci 26:2239-2256.

Southwell DG, Paredes MF, Galvao RP, Jones DL, Froemke RC, Sebe JY, Alfaro-Cervello C, Tang Y, Garcia-Verdugo JM, Rubenstein JL, Baraban SC, Alvarez-Buylla A (2012) Intrinsically determined cell death of developing cortical interneurons. Nature 491:109-113

Steadman PE, Ellegood J, Szulc KU, Turnbull DH, Joyner AL, Henkelman RM, Lerch JP (2014) Genetic effects on cerebellar structure across mouse models of autism using a magnetic resonance imaging atlas. Autism Res 7:124-137.

Stühmer T, Anderson SA, Ekker M, Rubenstein JL (2002) Ectopic expression of the Dlx genes induces glutamic acid decarboxylase and Dlx expression. Development 129:245-252.

Su H, Marcheva B, Meng S, Liang FA, Kohsaka A, Kobayashi Y, Xu AW, Bass J, Wang X (2010) Gamma-protocadherins regulate the functional integrity of hypothalamic feeding circuitry in mice. Dev Biol 339:38-50.

Sunkin SM, Ng L, Lau C, Dolbeare T, Gilbert TL, Thompson CL, Hawrylycz M, Dang C (2013) Allen Brain Atlas: an integrated spatio-temporal portal for exploring the central nervous system. Nucleic Acids Res 41:D996D1008.

Takeuchi O, Fisher J, Suh H, Harada H, Malynn BA, Korsmeyer SJ (2005) Essential role of BAX,BAK in B cell homeostasis and prevention of autoimmune disease. Proc Natl Acad Sci USA 102:11272-11277.

Taniguchi H (2014) Genetic dissection of GABAergic neural circuits in mouse neocortex. Front Cell Neurosci 8:8.

Taniguchi H, He M, Wu P, Kim S, Paik R, Sugino K, Kvitsiani D, Kvitsani D, Fu Y, Lu J, Lin Y, Miyoshi G, Shima Y, Fishell G, Nelson SB, Huang ZJ (2011) A resource of Cre driver lines for genetic targeting of GABAergic neurons in cerebral cortex. Neuron 71:995-1013.
Team RC (2018) R: a language and environment for statistical computing. In: $\mathrm{R}$ foundation for statistical computing. Available at https://www.Rproject.org/. Vienna: The R Foundation.

Thu CA, Chen WV, Rubinstein R, Chevee M, Wolcott HN, Felsovalyi KO, Tapia JC, Shapiro L, Honig B, Maniatis T (2014) Single-cell identity generated by combinatorial homophilic interactions between $\alpha, \beta$, and $\gamma$ protocadherins. Cell 158:1045-1059.

Vadodaria KC, Ji Y, Skime M, Paquola AC, Nelson T, Hall-Flavin D, Heard KJ, Fredlender C, Deng Y, Elkins J, Dani K, Le AT, Marchetto MC, Weinshilboum R, Gage FH (2019) Altered serotonergic circuitry in SSRIresistant major depressive disorder patient-derived neurons. Mol Psychiatry 24:808-818.

Verney C, Takahashi T, Bhide PG, Nowakowski RS, Caviness VS Jr (2000) Independent controls for neocortical neuron production and histogenetic cell death. Dev Neurosci 22:125-138.

Villalobos CA, Wu Q, Lee PH, May PJ, Basso MA (2018) Parvalbumin and GABA microcircuits in the mouse superior colliculus. Front Neural Circuits 12:35

Wagner-Golbs A, Luhmann HJ (2012) Activity-dependent survival of developing neocortical neurons depends on PI3K signalling. J Neurochem 120:495-501.

Wang Q, Shen FY, Zou R, Zheng JJ, Yu X, Wang YW (2017) Ketamineinduced apoptosis in the mouse cerebral cortex follows similar characteristic of physiological apoptosis and can be regulated by neuronal activity. Mol Brain 10:24.

Wang Q, Ding SL, Li Y, Royall J, Feng D, Lesnar P, Graddis N, Naeemi M, Facer B, Ho A, Dolbeare T, Blanchard B, Dee N, Wakeman W, Hirokawa KE, Szafer A, Sunkin SM, Oh SW, Bernard A, Phillips JW, et al. (2020) The Allen mouse brain common coordinate framework: a 3D reference atlas. Cell 181:936-953.e20.

Wang X, Weiner JA, Levi S, Craig AM, Bradley A, Sanes JR (2002) Gamma protocadherins are required for survival of spinal interneurons. Neuron 36:843-854.

Waters CM, Moser W, Walkinshaw G, Mitchell IJ (1994) Death of neurons in the neonatal rodent and primate globus pallidus occurs by a mechanism of apoptosis. Neuroscience 63:881-894.

Wei Y, Han X, Zhao C (2020) PDK1 regulates the survival of the developing cortical interneurons. Mol Brain 13:65.

Weiner JA, Wang X, Tapia JC, Sanes JR (2005) Gamma protocadherins are required for synaptic development in the spinal cord. Proc Natl Acad Sci USA 102:8-14.

Whyland KL, Slusarczyk AS, Bickford ME (2020) GABAergic cell types in the superficial layers of the mouse superior colliculus. J Comp Neurol 528:308-320.

Wong FK, Marín O (2019) Developmental cell death in the cerebral cortex. Annu Rev Cell Dev Biol 35:523-542.

Wong FK, Bercsenyi K, Sreenivasan V, Portalés A, Fernández-Otero M, Marín O (2018) Pyramidal cell regulation of interneuron survival sculpts cortical networks. Nature 557:668-673.

Wu Q, Maniatis T (1999) A striking organization of a large family of human neural cadherin-like cell adhesion genes. Cell 97:779-790.

Xu Q, Tam M, Anderson SA (2008) Fate mapping Nkx2.1-lineage cells in the mouse telencephalon. J Comp Neurol 506:16-29.

Xu S, Xiao Q, Cosmanescu F, Sergeeva AP, Yoo J, Lin Y, Katsamba PS, Ahlsen G, Kaufman J, Linaval NT, Lee PT, Bellen HJ, Shapiro L, Honig B, Tan L, Zipursky SL (2018) Interactions between the Ig-superfamily proteins DIP- $\alpha$ and Dpr6/10 regulate assembly of neural circuits. Neuron 100:1369-1384.e6.

Yamanaka H, Yanagawa Y, Obata K (2004) Development of stellate and basket cells and their apoptosis in mouse cerebellar cortex. Neurosci Res $50: 13-22$. 